This item was submitted to Loughborough's Research Repository by the author.

Items in Figshare are protected by copyright, with all rights reserved, unless otherwise indicated.

\title{
Simulation of swept-wing receptivity to distributed roughness
}

PLEASE CITE THE PUBLISHED VERSION

http://dx.doi.org/10.2514/6.2013-2842

\section{PUBLISHER}

Published by the American Institute of Aeronautics and Astronautics, Inc. @ the author(s)

\section{VERSION}

AM (Accepted Manuscript)

\section{PUBLISHER STATEMENT}

This work is made available according to the conditions of the Creative Commons Attribution-NonCommercialNoDerivatives 4.0 International (CC BY-NC-ND 4.0) licence. Full details of this licence are available at: https://creativecommons.org/licenses/by-nc-nd/4.0/

\section{LICENCE}

CC BY-NC-ND 4.0

\section{REPOSITORY RECORD}

Mistry, Vinan I., Gary J. Page, and James J. McGuirk. 2016. "Simulation of Swept-wing Receptivity to Distributed Roughness”. figshare. https://hdl.handle.net/2134/22502. 


\title{
Simulation of Swept-Wing Receptivity to Distributed Roughness
}

\author{
Vinan I. Mistry*, Gary J. Page ${ }^{\dagger}$ and James J. McGuirk ${ }^{\ddagger}$ \\ Loughborough University, Loughborough, Leicestershire, LE11 3TU, UK
}

\begin{abstract}
Simulations were carried out to model the receptivity and growth of stationary crossflow vortices from Distributed Roughness Elements (DRE) on a swept wing. A highly resolved Large Eddy Simulation (LES) numerical method was used for the study, the aim of the results were to achieve validation of the code to relevant experimental data and to gain a better understanding of the flow behaviour. The base flow for the simulations were based upon the experiment run by Hunt and Saric. ${ }^{1}$ The LES replicated the experimental setup using a WALE sub grid model and a streamline extraction process to only simulate the upper surface and reduce the overall computational expense. The WALE model is more suitable to modelling of transitional flows as it allows the sub-grid scale viscosity to vanish in laminar regions and in the inner regions of the boundary layer. Simulations were carried out for two spanwise wavelengths $(\lambda=6 \mathrm{~mm}, 12 \mathrm{~mm})$ and for roughness heights ranging from $12 \mu \mathrm{m}$ to $42 \mu \mathrm{m}$. The critical wavelength results showed, when comparing the stationary crossflow mode shapes, that the simulations at the larger roughness element sizes compare well with the experimental data. The control wavelength equally showed a good agreement to the experimental data at the larger roughness element sizes however the simulations over predict the amplitude of the smallest roughness element size. This can be attributed to either the simulation requiring a further refinement around the cylinder for the smallest roughness element size or to differences in the experimental and simulation roughness element shape. Overall the simulations successfully predict the receptivity from arrays of distributed roughness elements.
\end{abstract}

\section{Nomenclature}

TS Tollmien-Schlichting

CFL Courant Friedrichs Lewy Number

DRE Distributed Roughness Elements

$\Delta \quad$ Grid Filter

$\nu_{t} \quad$ Eddy Viscosity

$\nu \quad$ Kinematic Viscosity

$\mathrm{C}_{s} \quad$ Smagorinsky Constant

$\overline{S_{i j}} \quad$ Strain-Rate Tensor

$\mathrm{C}_{w} \quad$ WALE Constant

Q $\quad$ Freestream Velocity

c Chord

$\operatorname{Re}_{c} \quad$ Chord Reynolds Number

\section{Introduction}

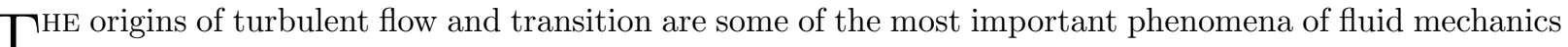
and aerodynamics and represents a challenging engineering problem for aircraft manufacturers looking

\footnotetext{
*Research Engineer, Email: V.I.Mistry@lboro.ac.uk

†Senior Lecturer, Email: G.J.Page@lboro.ac.uk

$\ddagger$ Professor of Aerodynamics, J.J.McGuirk@lboro.ac.uk
} 
to improve aerodynamic efficiency. With overall world passenger traffic expected to increase $4.7 \%$ per annum in the next decade ${ }^{2}$ the industry set ambitious targets to reduce $75 \%$ of carbon emissions from commercial aircraft, by 2050, relative to 2000 levels. ${ }^{3,4}$ A large part of the reduction will be achieved by innovative new engine configurations and the increased use of advanced materials, ${ }^{5}$ however the expected benefits of these technologies will not be sufficient. Reduction of aircraft drag by the delay of laminar-turbulent transition will be required to reduce the fuel burn and carbon emissions of future aircraft. Promoting laminar flow within a boundary layer has the potential for large savings in skin friction drag on aircraft. According to Schrauf $^{5}$ if the boundary layer flow on a wing was laminar on $30 \%$ of the surface area, the total drag of the aircraft could potentially be lowered by $12 \%$, a significant reduction.

Laminar flow is achieved on a civil aircraft wing by shifting the onset of transition from laminar to turbulent flow within the boundary layer as far aft as possible. This is done by manipulating the instabilities that cause the onset of transition. Three main instability mechanisms generally contribute to transition on a swept civil aircraft wing. The first is due to a combination of leading-edge radius and sweep which leads to the attachment-line mechanism; control of this instability is achieved by adjusting the attachment line Reynolds number $(\bar{R}<245){ }^{6,7}$ The second are streamwise instabilities that occur in the form of the familiar Tollmien-Schlichting (TS) waves that grow exponentially and lead to transition in the mid-chord region. It is now well known that shaping the airfoil for a favourable gradient and minimising the extent of the pressure-recovery region both contribute to the control of this instability. In regions of pressure gradient on highly swept surfaces the third mechanism, crossflow instability, causes transition close to the leading edge. The primary instability originates due to the combined influences of sweep and pressure gradient on an aircraft wing, generating curved streamlines at the boundary-layer edge. Curved streamlines generate centrifugal forces that are balanced by radial pressure gradients. Since static pressure can reasonably be assumed to be constant in the wall-normal direction within the boundary layer, the excess pressure due to its radial gradient at the boundary layer edge generates a mean cross-flow velocity. Because the crossflow velocity must vanish at the wall and at the edge of the boundary layer, an inflection point exists and provides the source of an inviscid instability. ${ }^{8,9}$

The crossflow instability exhibits amplified disturbances that can be stationary or travelling. Linear stability theory indicates that both stationary and travelling modes are unstable but that travelling waves are most amplified. However at low free stream turbulence conditions such as aircraft flight, stationary modes, excited from minute surface non-uniformity or roughness, have been found to be dominant with a stronger integrated disturbance. ${ }^{10,9}$ Deyhle and Bippes ${ }^{11}$ estimated background turbulence levels between $0.15 \%$ and $0.3 \%$ were necessary before travelling modes were the leading mechanism of laminar-turbulent transition. Crossflow vortices are also susceptible to a high frequency secondary instability that leads to rapid local breakdown, characterised by a turbulent wedge and saw-tooth pattern. ${ }^{12}$

A method for control of the crossflow instability was proposed by Saric et al. ${ }^{13}$ using a spanwise row of uniformly distributed roughness elements (DRE). Using artificial surface roughness in the form of the DRE's, a single mode is forced resulting in a smaller set of modes to measure and identify. Saric et al. ${ }^{13}$ demonstrated a delay in transition to turbulence using a DRE with micron-sized roughness elements and a wavelength spacing less than the most unstable wavelength (critical wavelength). The smaller wavelength (control wavelength) modifies the basic state such that the most unstable wavelength can no longer grow. The control wavelength then decays before amplitudes large enough to cause transition can occur. Malik et al. ${ }^{14}$ confirmed the stabilising effect of the subcritical disturbances by solving the nonlinear parabolized stability equations (NPSE) using the same base flow as Saric et al. ${ }^{13}$ Also using the same experiment Hunt \& Saric ${ }^{1}$ recently conducted a set of experimental receptivity tests to provide a database for numerical studies. They conducted tests at a critical and control wavelength at a number of micron sized roughness heights. Flight tests were conducted by Carpenter et al. ${ }^{15}$ demonstrating the capability of the method at higher chord based Reynolds number and the transition location moved from $30 \%$ chord to $60 \%$ chord.

Direct Numerical Simulation (DNS) is playing an increasingly important role in the investigation of crossflow transition thanks to the development of highly accurate discretisation methods and the advance in high performance computing. Various DNS work has been conducted by a number of research groups. Wasserman \& Kloker $^{12,16}$ conducted a spatial DNS study on a swept flat plate, they observed at critical wavelengths that the streaky nature of the stationary crossflow disturbance causes a strong mean flow distortion while the control cases reduced nonlinearly regions of deceleration within the steady mean flow. The strong deceleration favours the growth of secondary instabilities therefore Wasserman \& Kloker ${ }^{12,16}$ also concluded that the control mode not only stabilises the primary crossflow modes but also reduces the 
growth of the secondary instabilities.

Tempelmann et al. ${ }^{17}$ conducted a receptivity study using DNS and PSE and using results from an independent receptivity study using DRE's by Reibert et al. ${ }^{18}$ As the chord based Reynolds number for the experiment was $>2$ million the DNS was applied to the boundary layer region up to $70 \%$ chord with an initial RANS solution providing boundary conditions. Modal amplitudes extracted from DNS were $40 \%$ of that measured in the experiment however nonlinear PSE calculations revealed that the linear spatial evolution of the steady crossflow mode from the DNS and experiment were in good agreement, the discrepancy was attributed to additional receptivity in the experiments. Following on from this study Hosseini et al. ${ }^{19}$ used the same numerical method to perform a study using DRE's spaced at a control wavelength and a natural roughness case. They applied unsteady background disturbances using a weak randomly pulsed volume force and adjusted the amplitude until transition occurred at $45 \%$ chord for the natural roughness case. Using the same background disturbance transition was shown to move downstream using the control case.

The following paper describes results of a highly resolved Large Eddy Simulation (LES) to measure the receptivity from arrays of DRE's at different wavelengths and roughness heights. The base flow for the simulations was based upon the experiment run by Hunt and Saric. ${ }^{1}$ The experiment uses a ASU(67)-0315 aerofoil with a $45^{\circ}$ sweep angle and a chord based Reynolds number of 2.4 million. This flow configuration has been tested extensively by Saric and co-workers at Texas A\&M University and numerical work recently by the Henningson group at KTH Mechanics.

The results in this paper aim to replicate the experimental test setup and model two different spanwise wavelengths of DRE's and to measure the results against the experimental data. The numerical method in this paper is described as a highly resolved LES however the results remain laminar in the region of interest as no freestream perturbations are introduced. Therefore the main aim of the paper is to access the modelling requirements of the roughness arrays and to compare their receptivity to an experiment. Further work from this paper will introduce freestream perturbations and measure the impact on transition location. The overall flowfield and flow around the roughness elements will also be analysed to improve the current knowledge base of simulating distributed roughness.

\section{Numerical Method}

\section{A. Solver}

The computational code DELTA has been used for the LES and uses a finite volume pressure-based method on a multiblock structured grid. The code has been developed at Loughborough University and has been used for both RANS and LES calculations. ${ }^{20,21}$ The code uses a second order upwind scheme for spatial discretisation and a implicit backward Euler for temporal discretisation. Parallelisation is achieved by mapping the grid blocks to individual processors and updating the interface halo regions using message passing. ${ }^{22}$

LES simulations were run in a non-dimensional scheme based upon free stream velocity $Q_{0}$ and reference length, c. The time step for LES simulations was chosen such that the maximum CFL number was less than 1 , resulting in a non-dimensional time step for the grids used in this paper of $<1.0 \times 10^{-05}$. The solver was run until 10 non-dimensional flow through times had elapsed to allow the flow to develop; subsequently statistical averages were gathered by sampling to generate a time-averaged solution.

\section{B. Sub-Grid Modelling}

The success of LES for transitional flows is highly dependent on the underlying sub-grid scale (SGS) model used. The Smagorinsky model ${ }^{23}$ was used initially and assumes that the sub-grid scale viscosity is proportional to a characteristic sub-grid length $\Delta$ and to a characteristic turbulent velocity, which is taken as the product of $\Delta$ and the local resolved strain rate $|\bar{S}|$ magnitude, described below.

$$
\nu_{t}=\left(C_{s} \Delta\right)^{2}|\bar{S}|, \quad|\bar{S}|=\sqrt{2 \overline{S_{i j} S_{i j}}}
$$

An approximate value for $C_{s}$ is between $0.09-0.18$. The main problem with the Smagorinsky model is its behaviour near a wall. The Smagorinsky model generates a sub-grid scale viscosity wherever a velocity gradient exists, however, all turbulent fluctuations are damped near a wall, therefore $\nu_{t}$ should reduce to zero. A Van Driest ${ }^{24}$ exponential damping function can be applied along with reducing $C_{s}$ to 0.1 to sustain 
turbulence in channel flow; however this is an ad-hoc modification and is difficult to apply to a general case. Germano et al. ${ }^{25}$ proposed a dynamic Smagorinsky SGS model which adjusts the model coefficient to the local flow conditions e.g. reducing the model contribution near the wall in laminar and transitional regions. The dynamic model has showed promising results, however it is computationally expensive and difficult to implement for complex geometries. Results from this model also have to be clipped and adjusted for each case to provide a proper $y^{+3}$ near wall scaling for the eddy viscosity.

Nicoud \& Ducros ${ }^{26}$ subsequently proposed the Wall-Adapting Eddy Viscosity (WALE) model, that aims to recover the proper near wall scaling without a dynamic procedure and allows the eddy viscosity to tend to zero in laminar regions. The model is based on the square of the velocity gradient tensor and accounts for the effects of both the strain and rotation rate of the smallest resolved turbulent fluctuations. For the velocity gradient tensor $\bar{g}$ the model formulation is described below.

$$
\begin{gathered}
\nu_{t}=\left(C_{w} \Delta\right)^{2} \frac{\left(\mathscr{S}_{i j}^{d} \mathscr{S}_{i j}^{d}\right)^{\frac{3}{2}}}{\left(\bar{S}_{i j} \bar{S}_{i j}\right)^{\frac{5}{2}}+\left(\mathscr{S}_{i j}^{d} \mathscr{S}_{i j}^{d}\right)^{\frac{5}{4}}} \\
\mathscr{S}_{i j}^{d}=\frac{1}{2}\left(\bar{g}_{i j}^{2}+\bar{g}_{j i}^{2}\right)-\frac{1}{3} \delta_{i j} \bar{g}_{k k}^{2}, \quad \bar{g}_{i j}^{2}=\bar{g}_{i k} \bar{g}_{k j}
\end{gathered}
$$

Where $C_{w}$ is typically between $0.3-0.56$. Initially the Smagorinsky model was used to develop the flow and to stabilise the solution, subsequently the WALE model was used. For the simulations presented in this paper a value of 0.3 was used for $C_{w} \mathrm{~A}$ comparison of results obtained from the Smagorinsky and WALE model are detailed in Mistry et al. ${ }^{27}$

\section{Computational Models}

\section{A. Flow Configuration}

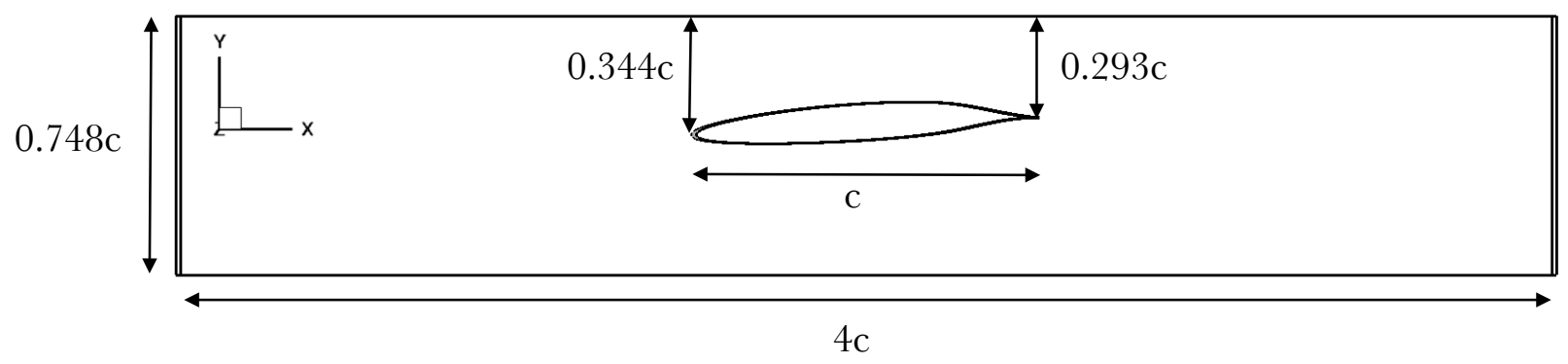

(a) Full Domain

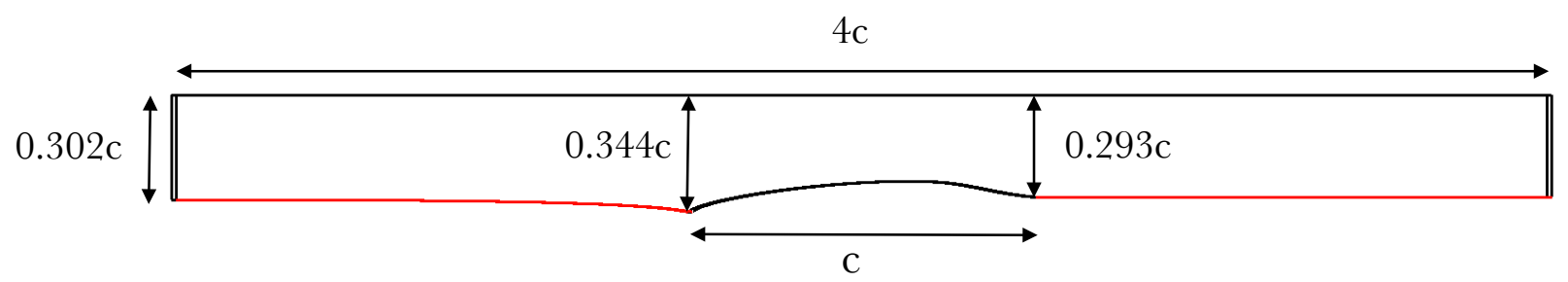

(b) Streamline Extracted Domain (Red lines show extracted streamlines)

Figure 1. Solution Domains

The base flow for the simulations was based upon the experiments of Hunt and Saric. ${ }^{1}$ The experiment used an ASU(67)-0315 aerofoil swept at an angle of $45^{\circ}$ and with an onset velocity $Q_{0}=22.5 \mathrm{~m} / \mathrm{s}$ giving a $R e_{c}$ of 2.4 million. The chord length $\mathrm{c}$ of the configuration was $1.83 \mathrm{~m}$, the wing was angled at $-2.9^{\circ}$ incidence and had a pressure minimum at $71 \% \mathrm{x} / \mathrm{c}$. The TS instability was stabilised by the favourable pressure gradient up to the pressure minimum, allowing crossflow to dominate boundary layer transition. 
Table 1. Table of roughness heights simulated

\begin{tabular}{lrr}
\hline \hline & Height k $(\mu m)$ & Spanwise Wavelength $\lambda(\mathrm{mm})$ \\
\hline Case 1a & 14 & 6 \\
Case 1b & 27 & 6 \\
Case 1c & 42 & 6 \\
Case 2a & 12 & 12 \\
Case 2b & 24 & 12 \\
Case 2c & 36 & 12 \\
\hline
\end{tabular}

The experiments used cylindrical shaped roughness elements for their DRE and were placed at $2.9 \% \mathrm{x} / \mathrm{c}$ near the crossflow neutral point to ensure uniform disturbances and a cylinder diameter of $3 \mathrm{~mm}$. Several configurations of the roughness arrays were used and designated with the notation $[\mathrm{k}, \lambda]$, where $\mathrm{k}$ is the amplitude of the roughness in microns and $\lambda$ is the spanwise wavelength in millimetres. The wavelength is measured from the centre of each roughness element. Hunt and Saric ${ }^{1}$ used two $\lambda$ spacings. The first was termed the critical wavelength $\lambda$ at $12 \mathrm{~mm}$ and the second was the control wavelength $\lambda$ at $6 \mathrm{~mm}$. The experiments were conducted at several roughness heights, the cases that were run in this paper are detailed in Table 1. Hot-wire measurements were taken at $10 \%, 15 \%$ and $20 \% x / c$ to measure the receptivity and growth rate of the disturbances.

The initial solution domain for the computational model is shown in Figure 1(a). The (x-y) plane dimensions of the domain were kept similar to that of the wind tunnel in the experiment with $0.748 c$ in the transverse direction, a length of $4 c$ was used in the streamwise direction. The spanwise dimension is an important quantity for the LES; a large enough segment is required to fully simulate the spanwise array, however a too large segment requires a much greater computational expense. To reduce the impact of the spanwise faces an infinite swept wing model was applied using a periodic or cyclic condition on the spanwise faces. This is achieved by linking the topology of each spanwise face from each block, allowing data to be passed from one side face back into the block on the opposite face. DNS studies ${ }^{17}$ showed that using two roughness elements was adequate for modelling a spanwise cyclic roughness array. A spanwise segment (parallel to the leading edge) of $24 \mathrm{~mm}$ was used for all cases. The upper bound of the solution domain was modelled as a symmetry boundary condition.

To reduce the computational expense of the simulation only the upper surface of the solution domain was simulated. This was achieved by exporting the stagnation streamline upstream of the leading edge and the streamline aft of the trailing edge from the steady, time averaged mean solution of an initial coarse LES of the full domain. These were averaged across the span and converted (together with the geometry of the upper airfoil surface) into a plane which defined the inner boundary of a new solution domain. Figure 1(b) shows the streamline extracted domain. Pressure and velocity results taken from the streamline extracted domain showed good agreement with the full domain, showed in Figure 6.

\section{B. Grids}

An initial grid and a modified grid were generated using ICEMCFD 14.0. For both grids a C-grid was place around the aerofoil to ensure good quality cells in the boundary layer region. Blocks were then extended towards the inlet and outlet from the C-grid. To mesh the circular cylinders on the wing surface an O-grid was place above each cylinder, Figure 2(a) shows the O-grid blocking structure around each cylinder and Figure 2(b) shows a slice of the modified grid in the z-plane across the a cylinder.

The grid diagnostics for each grid are listed in Table 2. Piomelli \& Balaras ${ }^{28}$ advise on the necessary grid requirements for resolving a turbulent boundary layer with near-wall streaks using LES and the both initial and modified grids are within the criteria described (Streamwise $\Delta x+\lesssim 100$ and Spanwise $\Delta z+\lesssim 20$ ). The initial grid was developed to judge the grid resolution requirements necessary to capture the initial amplitude of the disturbance and the spacing required to capture the development and growth of the crossflow vortices. The initial grid consists of 2137 nodes in the streamwise direction and a $\Delta x+=15$ near the cylinder. 70 nodes in the wall normal direction capture the boundary layer with a $\Delta y+=0.5$ at the wall. In the spanwise direction 100 nodes are placed (parallel to the leading edge) with a $\Delta z+=16$. Figure 3(a) shows the mesh resolution around the cylinder for the initial grid. No extra refinement is placed around the cylinder. 
The modified grid was developed when results from the initial grid were obtained. Extra refinement was placed around the cylinder to capture the disturbance near the wall. The grid spacing was decreased in all directions, 2550 nodes in the streamwise direction $(\Delta x+=8$ near the cylinder $), 100$ nodes in the wall normal boundary layer region $(\Delta y+=0.25$ at the wall) and 156 nodes in the spanwise direction $(\Delta z+=11)$.

Table 2. Grid Diagnostics

\begin{tabular}{lrr}
\hline \hline & Initial Grid & Modified Grid \\
\hline Block Count & 142 & 190 \\
Airfoil Nodes & 2137 & 2550 \\
BL Wall Normal Nodes & 70 & 100 \\
Spanwise Nodes & 100 & 156 \\
Leading Edge, $\Delta x+$ & 40 & 30 \\
Cylinder, $\Delta x+$ & 15 & 8 \\
Trailing Edge, $\Delta x+$ & 60 & 60 \\
$\Delta y+$ & 0.5 & 0.25 \\
$\Delta z+$ & 16 & 11 \\
Total Mesh Size & $60 \mathrm{mil}$ & $95 \mathrm{mil}$ \\
\hline
\end{tabular}

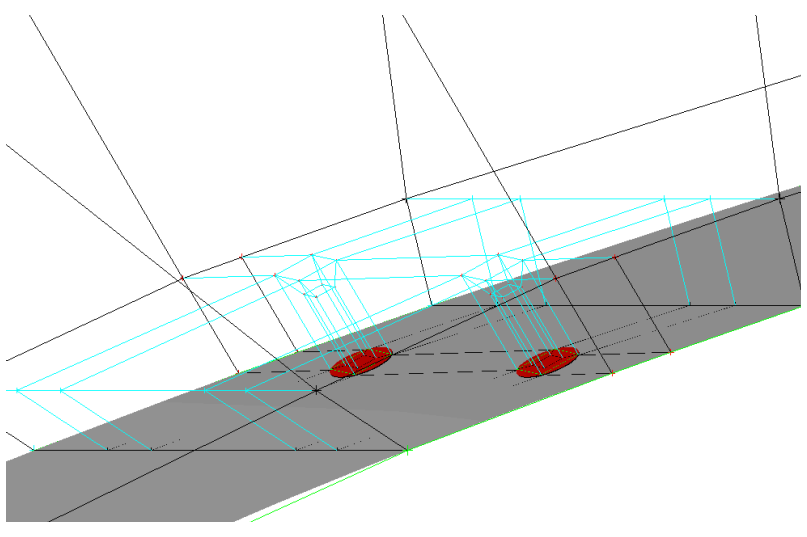

(a) Modified grid cylinder blocking structure

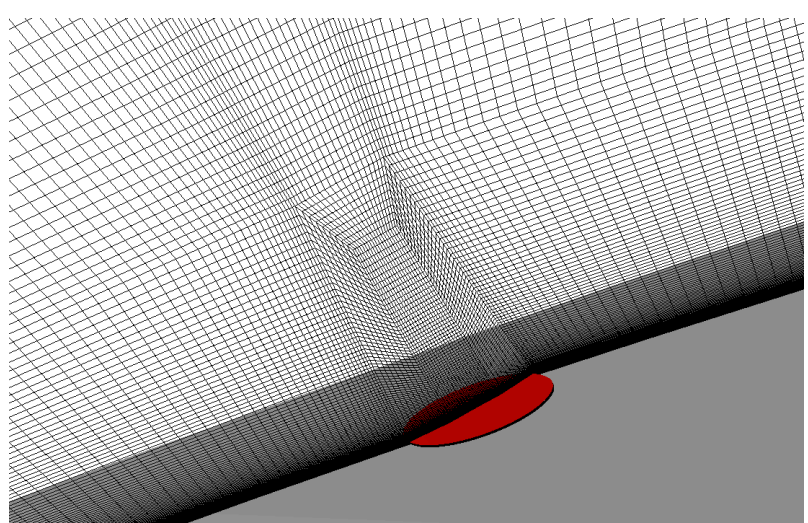

(b) Slice of modified grid through cylinder

Figure 2. Mesh spacing around cylinder

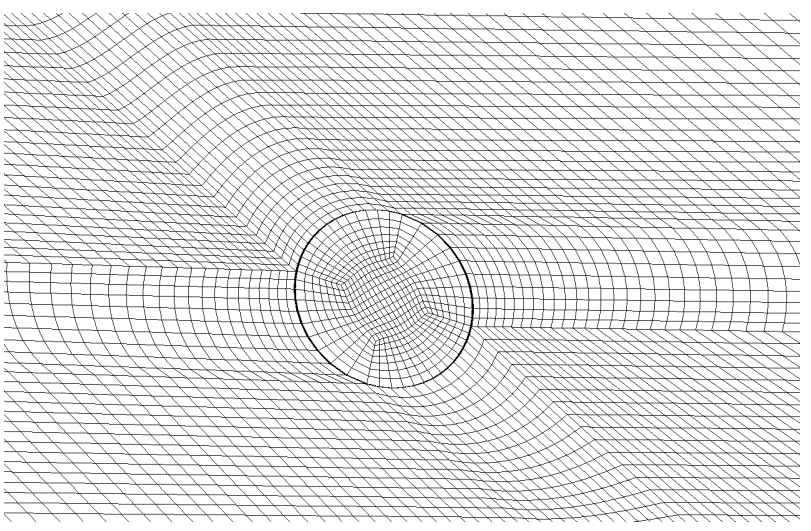

(a) Initial Grid

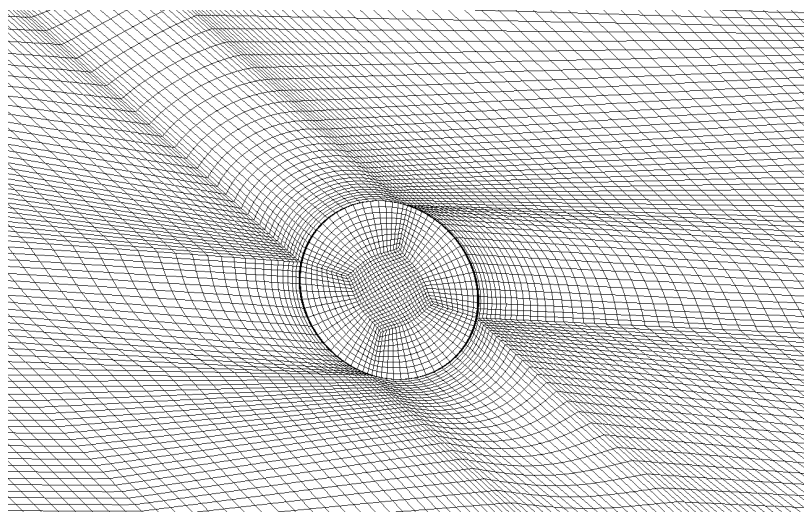

(b) Modified Grid

Figure 3. Grids 


\section{Impact of Grid Spacing}

The velocity results described in this paper are aligned with the global cartesian coordinate system shown in Figure 1(a). The $u$ velocity is aligned with the global $\mathrm{x}$-coordinate in the streamwise direction and the $w$ velocity is aligned with the global z-coordinate in the spanwise direction (Note: The $w$ velocity component is not parallel to leading edge of the wing, but parallel to the unswept z-coordinate).

Both grids were run on a single case to demonstrate the impact of the grid spacing on the results. Figure 4 shows contours of spanwise velocity around the cylinder for both initial and modified grid. The slice is taken at the upper edge of the cylinder with the flow from left to right. The contour shows acceleration of the spanwise velocity component on each side of the cylinder. The area of acceleration can be shown to extend $1 / 5$ of the cylinder diameter away from the cylinder. For the initial grid only 3-5 cells capture this acceleration around the cylinder, resulting in the simulation poorly capturing the initial disturbance amplitude. The modified grid was developed to capture this region better with 8-10 cells capturing the acceleration of flow around the cylinder. The impact that the poor resolution of the cylinder in the initial grid can be shown in Figure 5. The figure shows a stationary mode shape for Case $2 \mathrm{~b}(\lambda=12 \mathrm{~mm}, \mathrm{k}=$ $24 \mu \mathrm{m})$ at $15 \%$ chord. The calculation of the mode shape is explained in Section V. The initial grid under predicts the amplitudes while the modified grid matches very well with the experiment. This suggests that the emphasis must be placed on the cells nearest to the cylinder and to fully capture the initial amplitude. Subsequently, the modified grid was used for the remaining simulations and the results presented in the following sections are from the modified grid.

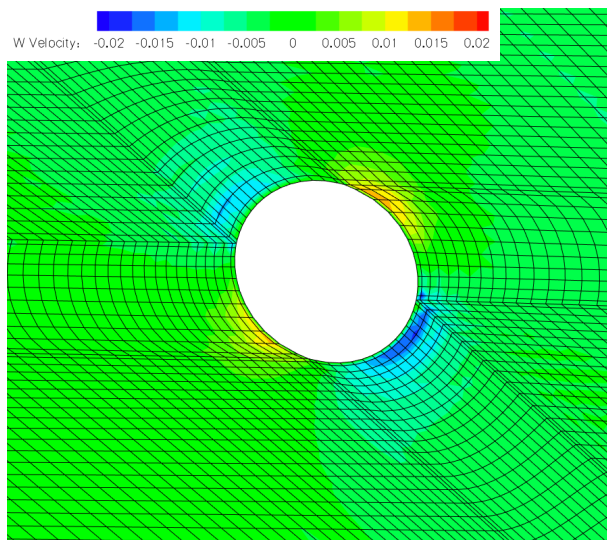

(a) Initial Grid

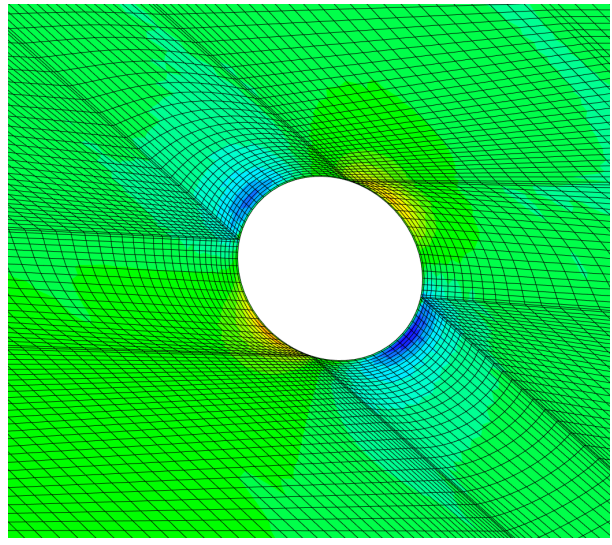

(b) Modified Grid

Figure 4. $k=24 \mu m, \lambda=12 \mathrm{~mm}$ Contours of spanwise velocity at cylinder height $\left(w / Q_{0}\right)$, top view at cylinder edge, Flow: Left to Right

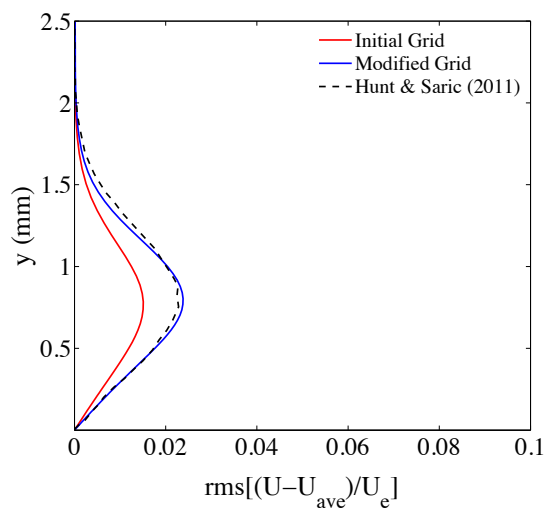

Figure 5. $k=24 \mu m, \lambda=12 \mathrm{~mm}$, Stationary mode shape for initial grid, modified grid and experimental results at $15 \% x / c$ 


\section{Base Flow Results}

\section{A. Laminar Base Flow}

The base flow of the LES results were validated against the experimental results available from Hunt and Saric. ${ }^{1}$ The pressure coefficients extracted from the LES are compared to the experimental results in Figure 6(a). The figure shows a curve for the full domain and the streamline extracted domain described in Figure 1. The streamline extracted domain pressures match very well with the full domain demonstrating the success of the approach. The experimental pressure coefficient results are presented at two locations, the wing was mounted vertically in the wind tunnel and pressure measurements were taken on the upper and lower part of the span to ensure spanwise uniformity. The results from the LES show very good agreement with the experimental pressure measurements. The actual pressure is slightly higher than the experimental results in the favourable pressure gradient region leading to the pressure minimum, however, the pressure gradient is well matched. As the pressure gradient is the feature that effects boundary layer transition and stability the LES is considered to be suitably matched to the experiment.

Figure 6(b) shows a boundary layer profile for the streamwise $(\mathrm{u})$ velocity component at $10 \% \mathrm{x} / \mathrm{c}$ for both the LES and the experimental results. The graph shows an excellent agreement between the simulation and experimental profiles.

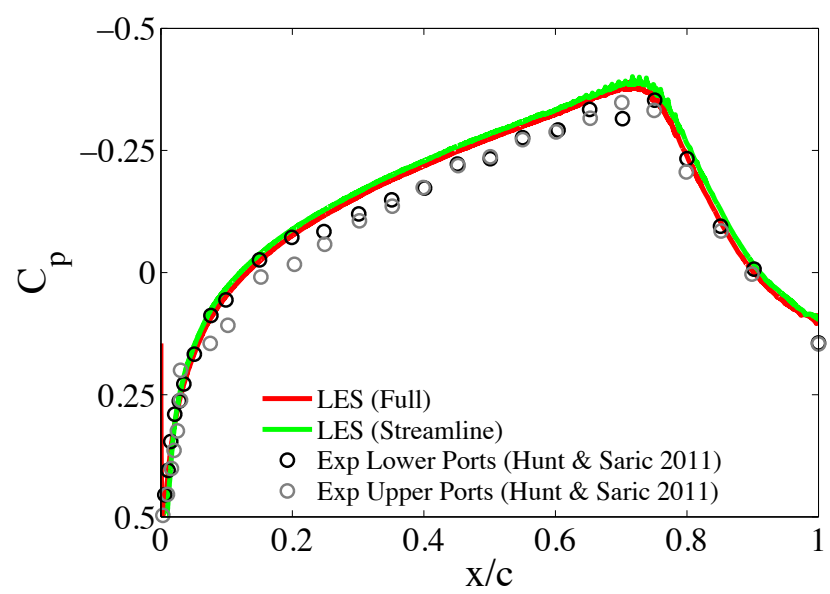

(a) Pressure Coefficient

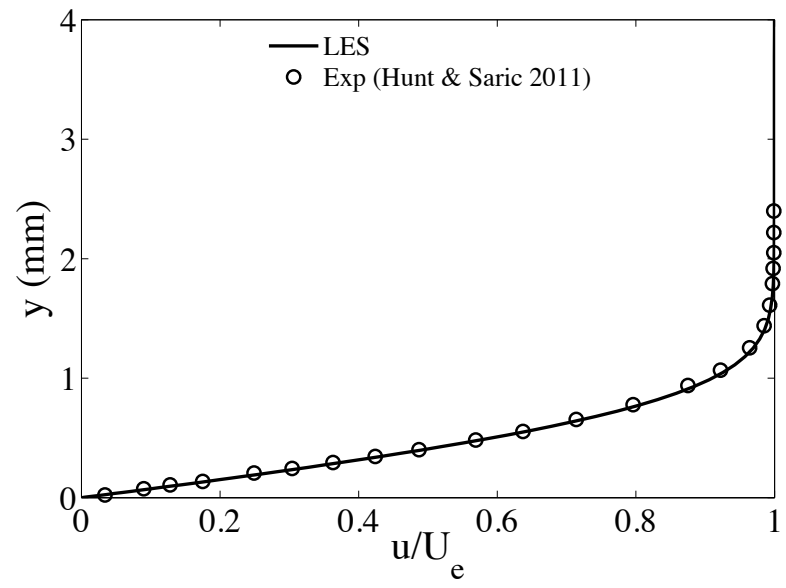

(b) Streamwise $(\mathrm{u})$ boundary layer profile at $10 \% \mathrm{x} / \mathrm{c}$

Figure 6. Comparison of Laminar Base Flow from LES to Experimental 


\section{B. Flow around Cylinder}

Simulation of the array of micron-sized cylinders were carried out once the laminar base flow was validated. The cylinders disturb the laminar base flow by accelerating the flow around the cylinders, illustrated in Figure 4 by contours of spanwise velocity $\left(w / Q_{0}\right)$. The figure shows a disturbance velocity of $0.02 w / Q_{0}$ of opposite sign each side of the cylinder. This disturbance can be visualised in Figure 7 in the form of streamlines. Figure 7(a) shows streamlines close up to the cylinder with the streamlines following the shape of the cylinder. Figure 7(b) shows the disturbance generated for the roughness array and beginning to develop downstream of the roughness element array.

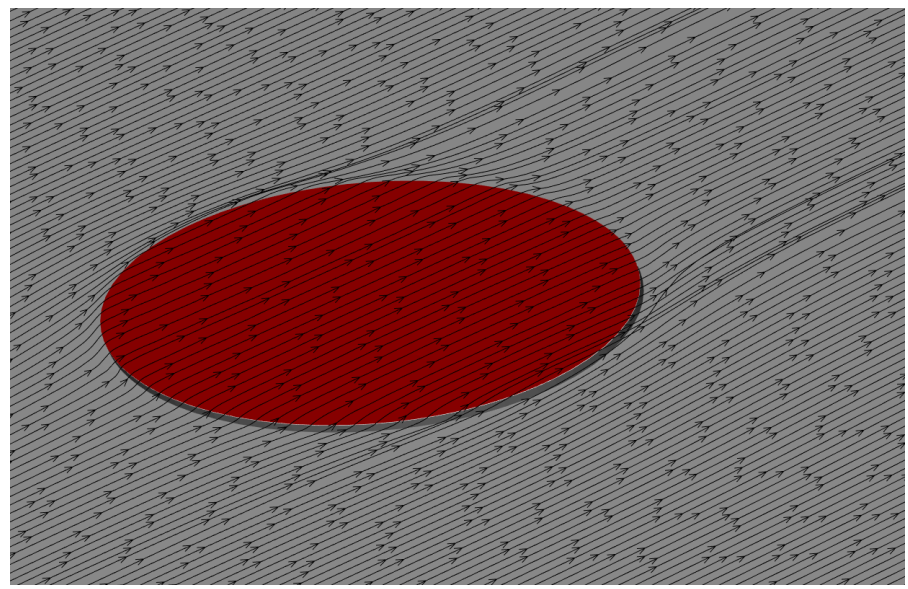

(a) Streamlines at cylinder height edge, $k=36 \mu \mathrm{m}, \lambda=12 \mathrm{~mm}$ (Isometric view)

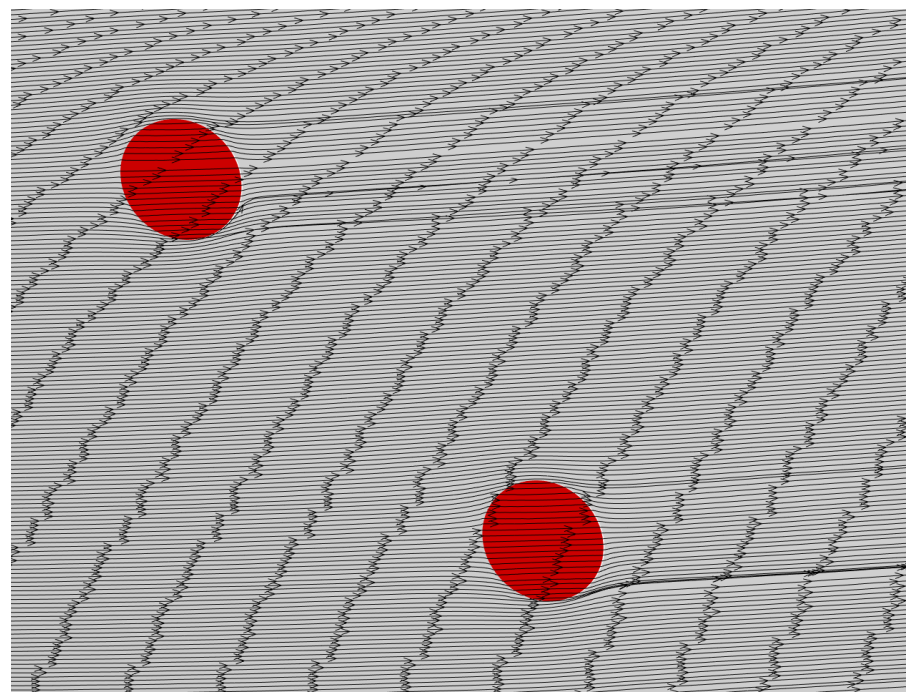

(b) Streamlines at cylinder height edge, $k=36 \mu m, \lambda=12 m m$ (Top view)

Figure 7. Streamlines around cylindrical roughness element 


\section{Receptivity of DRE Array at Critical Wavelength Results}

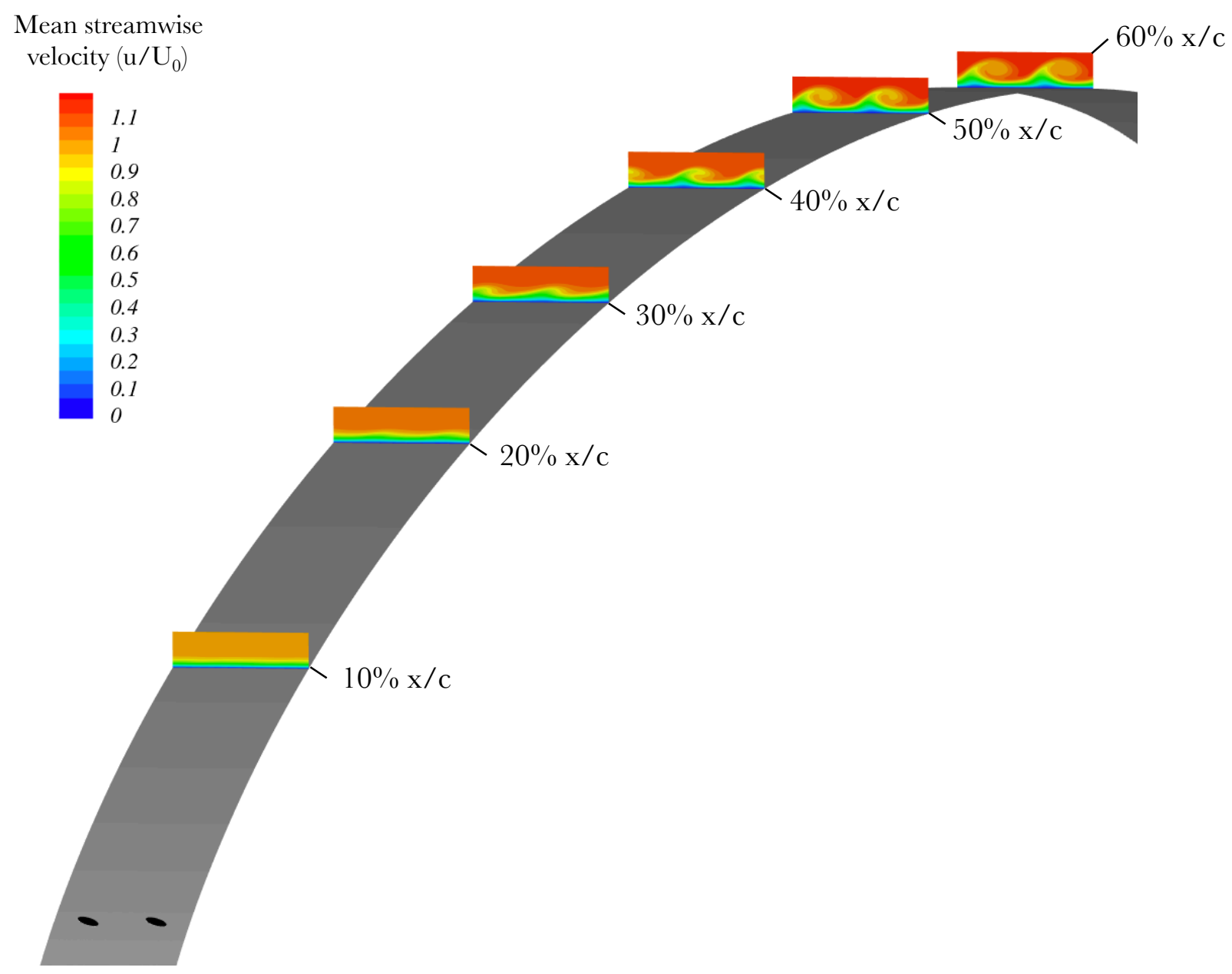

Figure 8. Contours of mean streamwise velocity $u / U_{0}, k=36 \mu m, \lambda=12 \mathrm{~mm}$, Contours taken at $x / c=$ $10 \%, 20 \%, 30 \%, 40 \%, 50 \%, 60 \%$

Simulations were run initially for the critical $(\lambda=12 \mathrm{~mm})$ wavelength for the cylindrical roughness heights described in Table 1. An overview of the flowfield is shown in Figure 8 for the the $k=36 \mu m$ case. The figure shows contours of streamwise velocity and the spatial development of the crossflow vortices. The disturbance and spanwise variation of the flow field becomes apparent from $20 \% x / c$ with the disturbance growing rapidly and the formation of two distinct crossflow vortex formations at $40 \% x / c$. Also evident from the contour at $40 \% x / c$ is the rollover effect, the high momentum fluid overlapping the low momentum fluid. The overall development, shape and size of the crossflow vortex conforms with descriptions and images from previous literature using the same base flow. ${ }^{1,17,18}$

The experimental test case measured the receptivity of the roughness element arrays by applying hotwire measurement scans at $15 \%$ and $20 \% x / c$. The process to determine the amplitude and stationary mode shape for each roughness element is replicated for the LES to measure the accuracy of the method.

Figure 9 shows contours of mean flow streamwise velocity $\left(u / U_{e}\right)$ at $15 \% x / c$ for the three roughness element heights. The figures on the left show the experimental hot-wire scans while the figures on the right show the simulation results. The hot-wire scans were taken with a $1 \mathrm{~mm}$ spacing resulting in 65 locations across the span. The simulation however contains 156 spanwise locations across $24 \mathrm{~mm}$ and the additional detail can be shown in the contours. The contours show a clear $12 \mathrm{~mm}$ periodicity in the results for both the experiments and simulations. The increase in disturbance amplitude for the three roughness heights can also be clearly visualised for both the experiments and simulations.

Figure 10 shows mean flow streamwise velocity profiles taken from the contours shown in Figure 9. The 
mean boundary layer profile is displayed in the figure and coloured red. The mean velocity profile does not represent the base flow in absence of roughness element, but rather, the disturbed flow downstream of the roughness array. The range of velocity profiles for the simulations match well with the experimental profiles.

Figure 11 shows the disturbance velocity profiles. These figures are obtained by calculating a mean of the individual velocity profiles (shown in red in Figure 10) and mousing from each individual velocity profiles in the span. The disturbance profiles make it much easier to show how the roughness height increases the disturbance and deviation away from the mean velocity profile. The simulation figures shown on the right display a good resemblance to the experimental data. The main deviation from the experimental results are in the positive disturbance, the simulation results show a stronger positive disturbance compared to the experiment, particularly for the $12 \mu \mathrm{m}$ case. The increased positive disturbance is balanced from the LES by less negative disturbance. A possible reason for this imbalance is that more individual profiles are taken to calculate the mean profile, resulting in more symmetrical disturbance profiles.

The stationary mode shape can be calculated by taking the root-mean-square of the disturbance profiles, this shows the total disturbance amplitude of all modes at the streamwise location. Figure 12(a) shows stationary mode shapes for each roughness element at 15\% x/c and Figure 13 (a) at 20\% $x / c$.

At $15 \% x / c$ the stationary mode shapes show a single lobe with a maximum amplitude at approximately $0.75 \mathrm{~mm}$ from the wall. The stationary modes show a linear growth between each roughness height. The simulations for the larger roughness height show an excellent agreement with the experimental profile. For the $k=36 \mu m$ and $k=24 \mu m$ cases the profiles lie very close to the experimental curve. However at $k=12 \mu \mathrm{m}$ the amplitude of the disturbance for the simulation is approximately $25 \%$ greater than that of the experiment. The cause of the additional receptivity in the simulations is unclear but as the $k=12 \mu m$ is the smallest roughness height used the resolution of the grid may be insufficient. Also at this roughness height the experimental geometry showed a substantial standard deviation $(\sim 2 \mu m)$ in the roughness height which may also impact the receptivity.

The stationary mode shape is a representative of the total disturbance signal which may contain multiple modes. Using the boundary layer height at which the disturbance is at its maximum the mode shape can be spatially decomposed into its modal amplitudes by taking a Fast Fourier Transform (FFT) of the signal. Figure 12(b) shows a plot of amplitude against wave number for a spanwise signal at maximum amplitude $(0.75 \mathrm{~mm})$ at $15 \% \mathrm{x} / \mathrm{c}$. The figure shows predominant modal amplitudes at $12 \mathrm{~mm}$ and $6 \mathrm{~mm}$ at $15 \% \mathrm{x} / \mathrm{c}$.

At $20 \% x / c$ the stationary mode shapes remain with a single disturbance lobe and a greater distance between amplitudes for each roughness height. The $k=12 \mu \mathrm{m}$ and $k=24 \mu \mathrm{m}$ cases match extremely well with the experiment, however, the $k=36 \mu \mathrm{m}$ case under predicts the maximum amplitude of the disturbance compared to the experiment. The difference in amplitude is approximately 15\%. Figure 13(b) shows a amplitude spectra plot for a spanwise signal at maximum amplitude $(0.9 \mathrm{~mm})$ at $20 \% x / \mathrm{c}$. The fundamental $12 \mathrm{~mm}$ mode is the predominant wave number while the $6 \mathrm{~mm}$ mode remains stable. 
Experiment

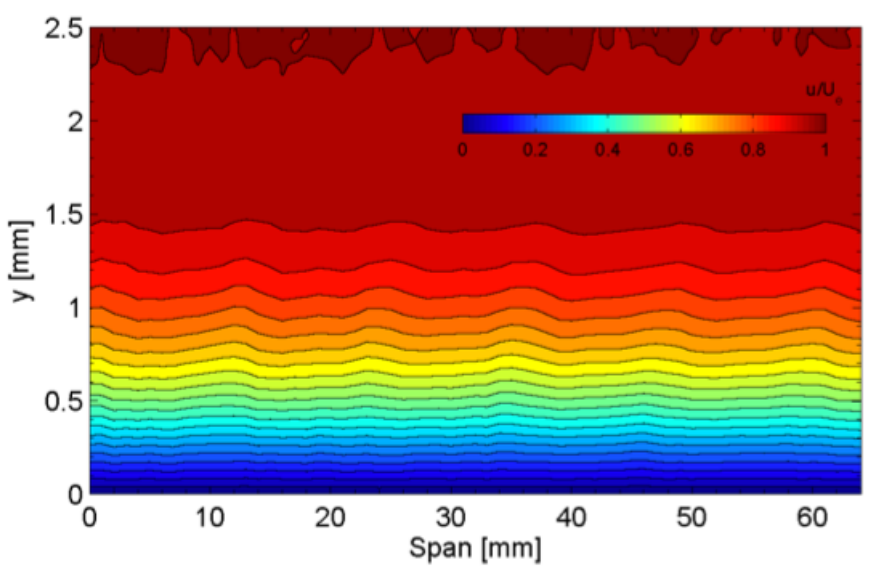

(a) $k=12 \mu m, \lambda=12 m m$

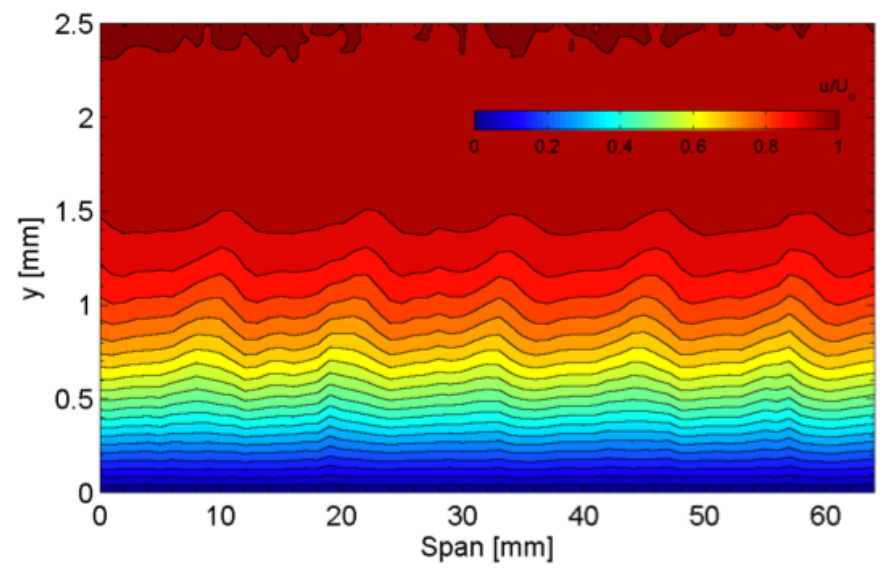

(b) $k=24 \mu m, \lambda=12 m m$

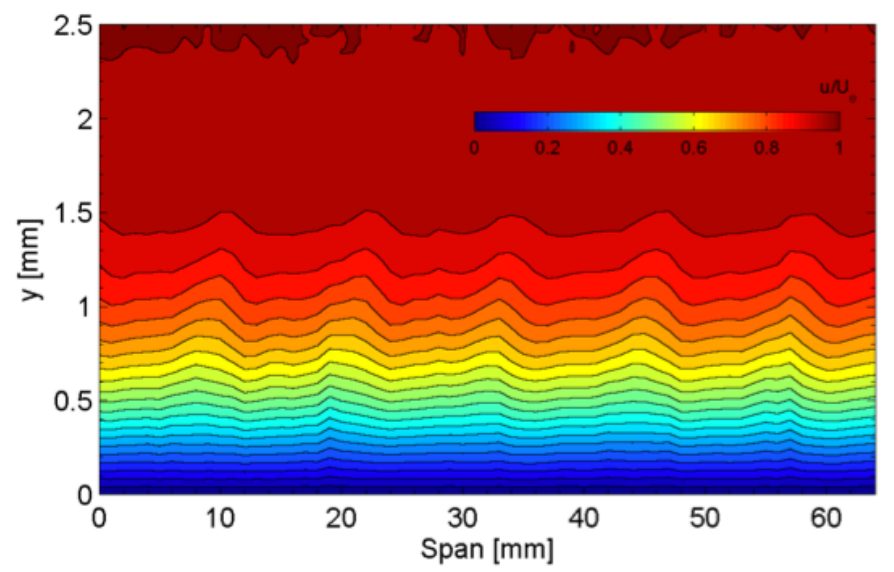

(c) $k=36 \mu m, \lambda=12 m m$
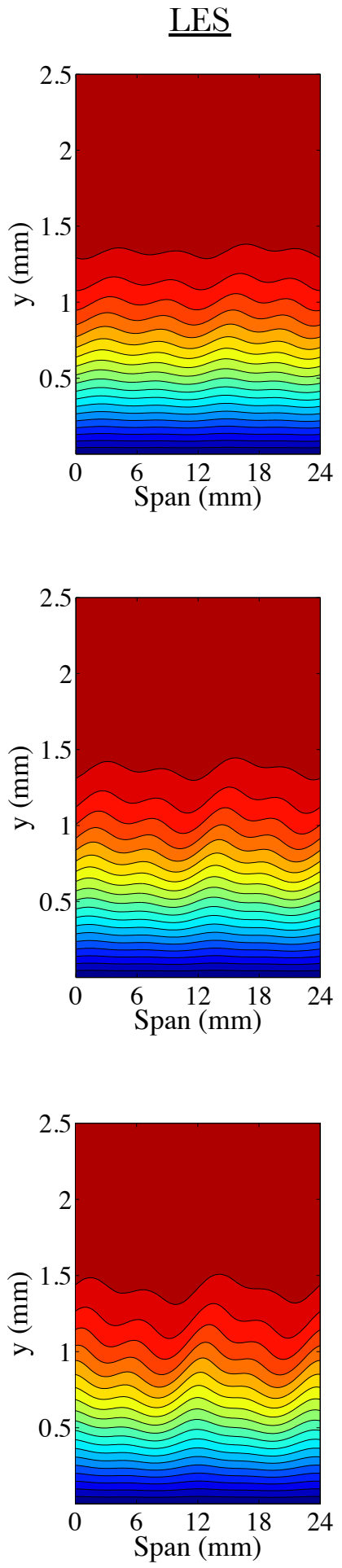

Figure 9. Contours of streamwise velocity $\left(u / U_{e}\right)$ at $15 \% x / c$ Left: Experimental (Hunt and Saric ${ }^{1}$ ) Right: LES 
$\underline{\text { Experiment }}$

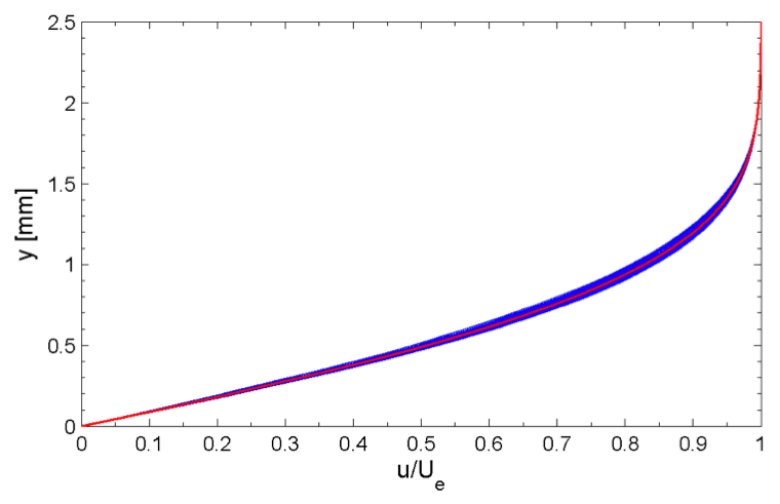

$\underline{\text { LES }}$

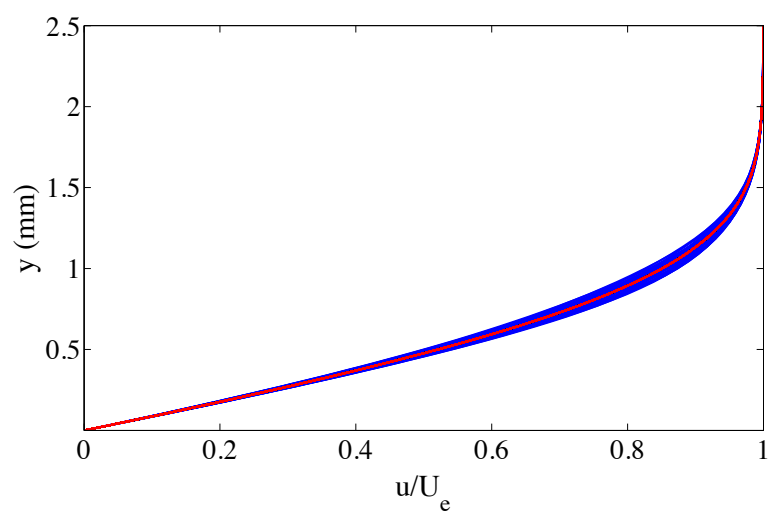

(a) $k=12 \mu m, \lambda=12 m m$
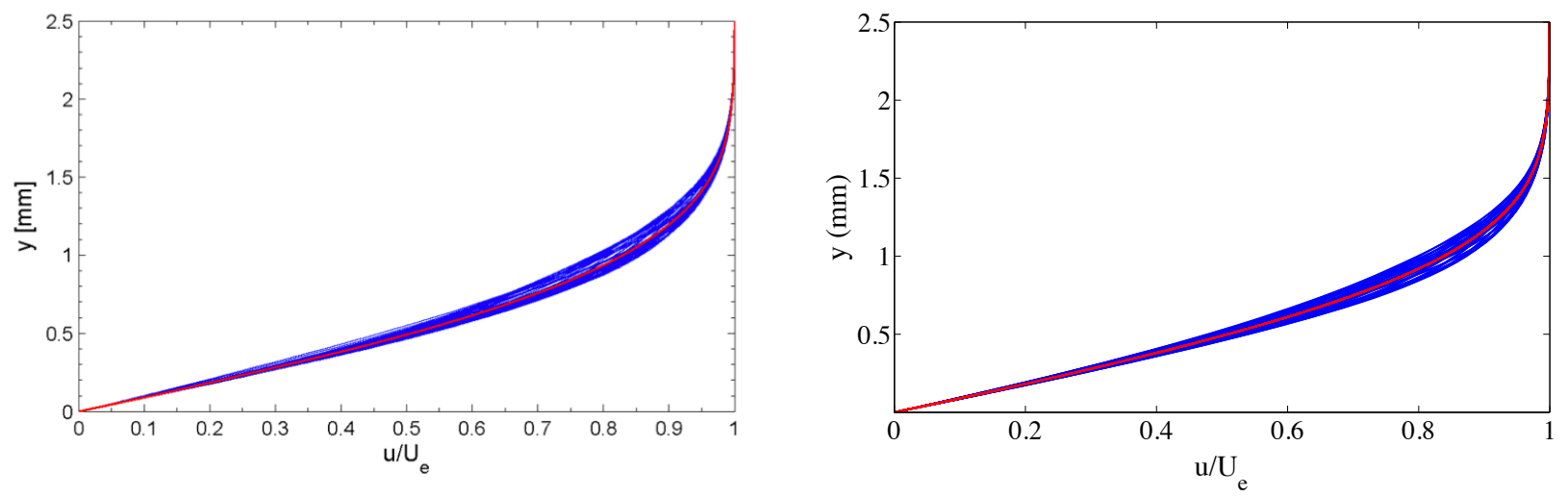

(b) $k=24 \mu m, \lambda=12 m m$
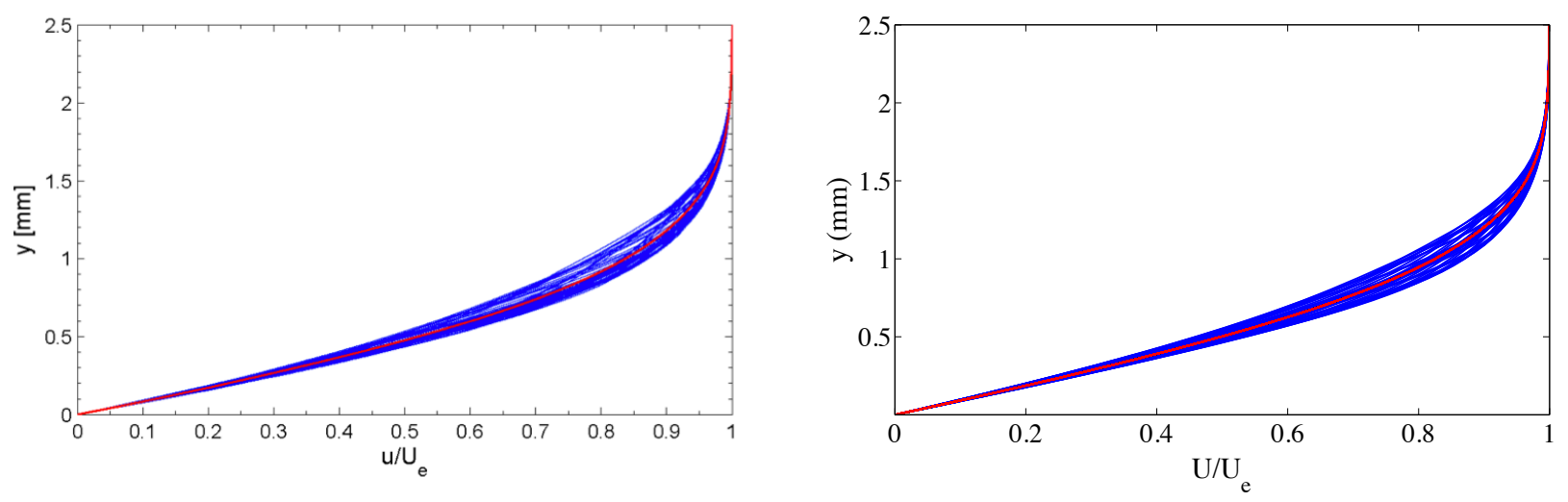

(c) $k=36 \mu m, \lambda=12 m m$

Figure 10. Spanwise array of mean-flow boundary-layer profiles across span at $15 \% x / c$, The mean of the profiles is displayed in red, Left: Experimental (65mm span) (Hunt and Saric ${ }^{1}$ ) Right: LES (24mm span) 

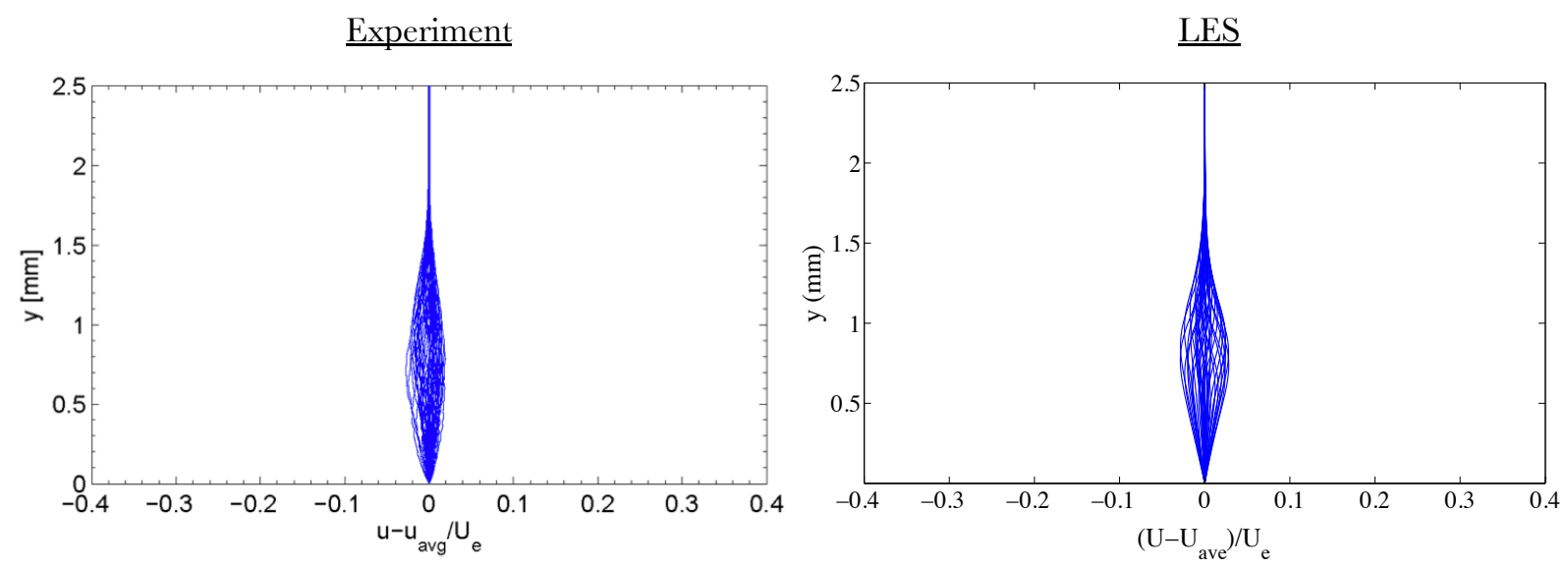

(a) $k=12 \mu m, \lambda=12 m m$
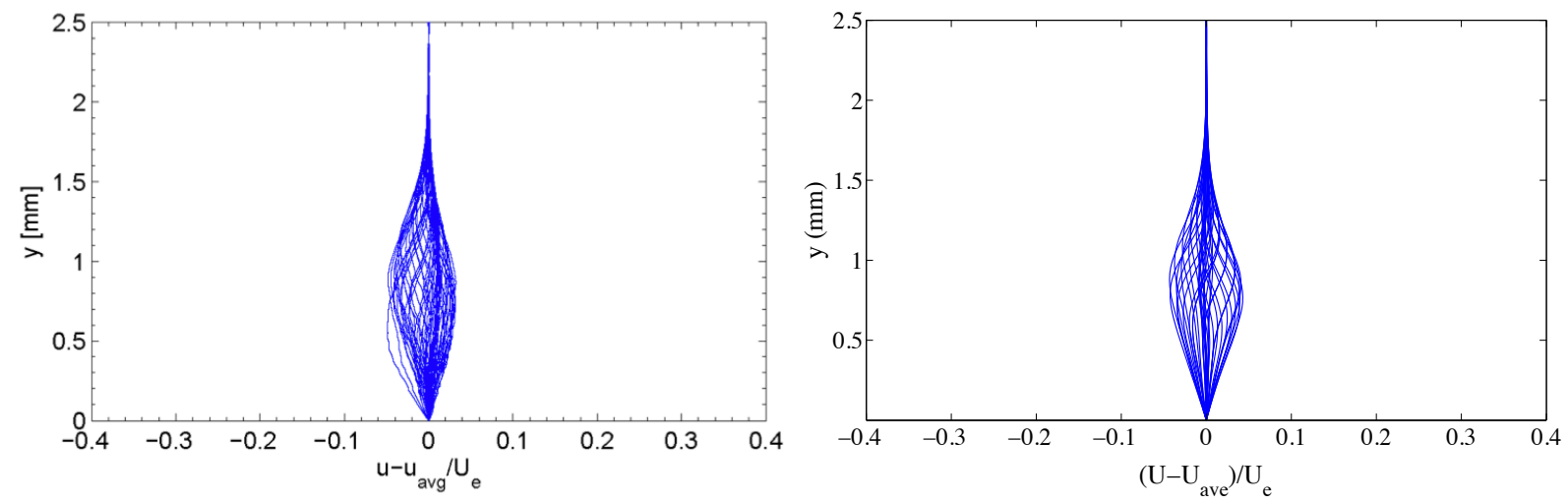

(b) $k=24 \mu m, \lambda=12 m m$
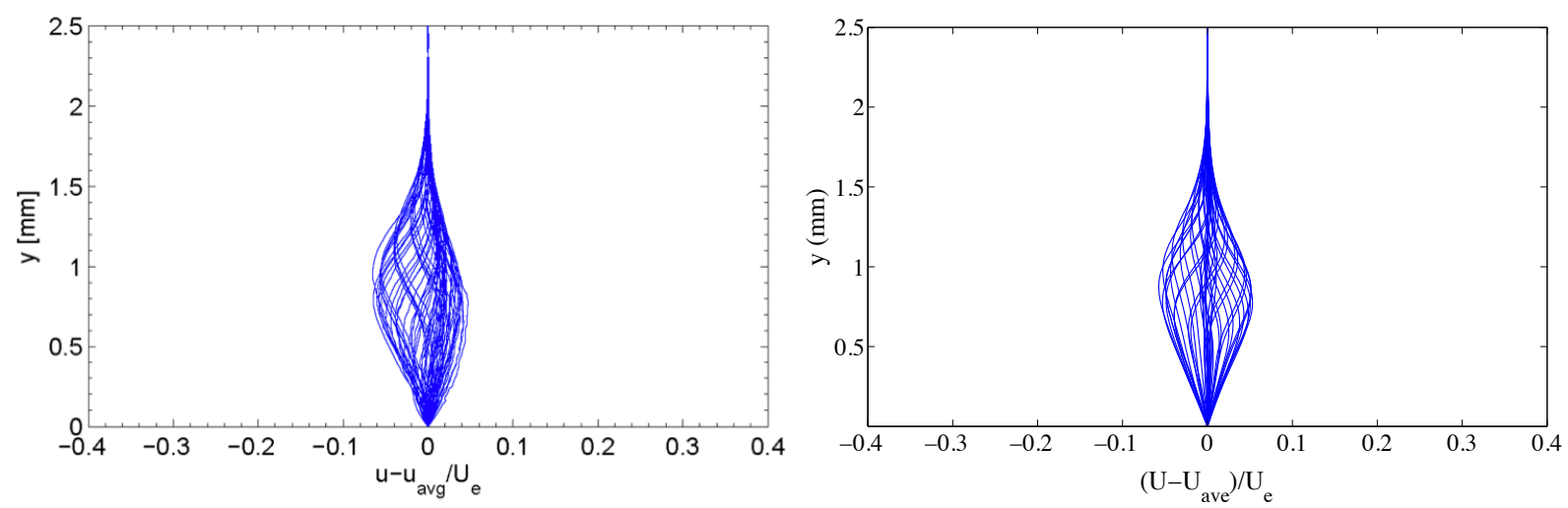

(c) $k=36 \mu m, \lambda=12 m m$

Figure 11. Spanwise array of disturbance velocity profiles across span at $15 \% x / c$ Left: Experimental (65mm span) (Hunt and Saric ${ }^{1}$ ) Right: LES (24mm span) 


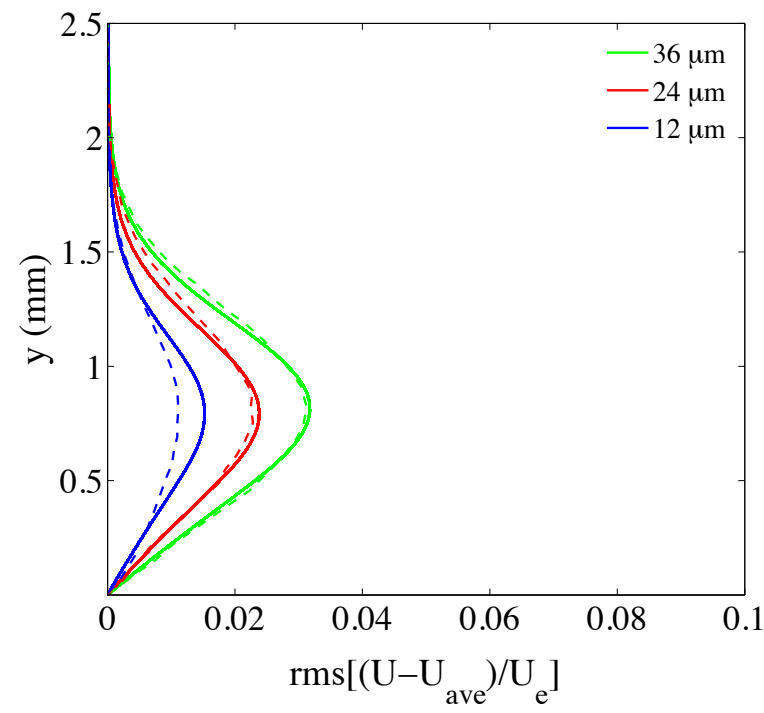

(a) Stationary crossflow mode shape

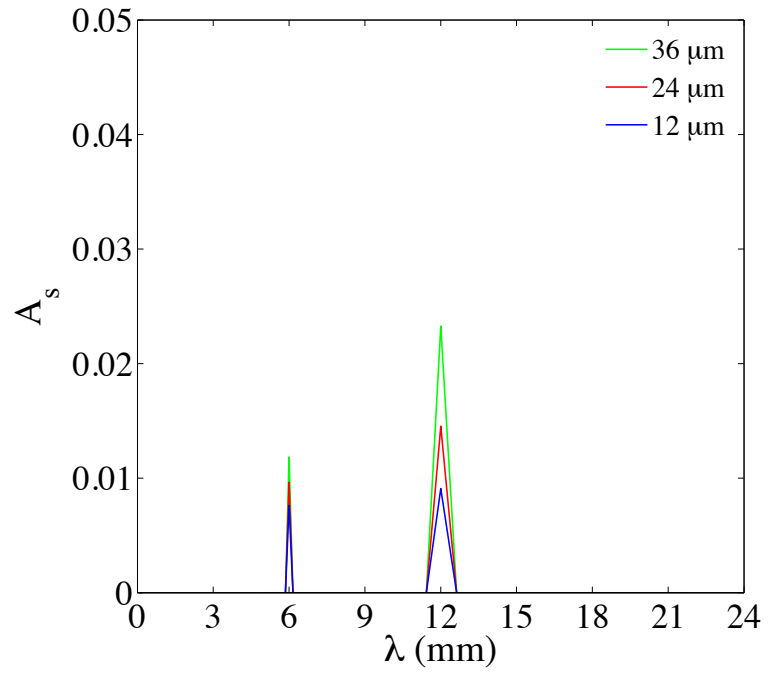

(b) Amplitude vs wave number for spanwise signal at maximum amplitude

Figure 12. Stationary mode shape and amplitude spectra at $15 \% x / c, \lambda=12 \mathrm{~mm}$ (Roughness height, k, indicated on graph)

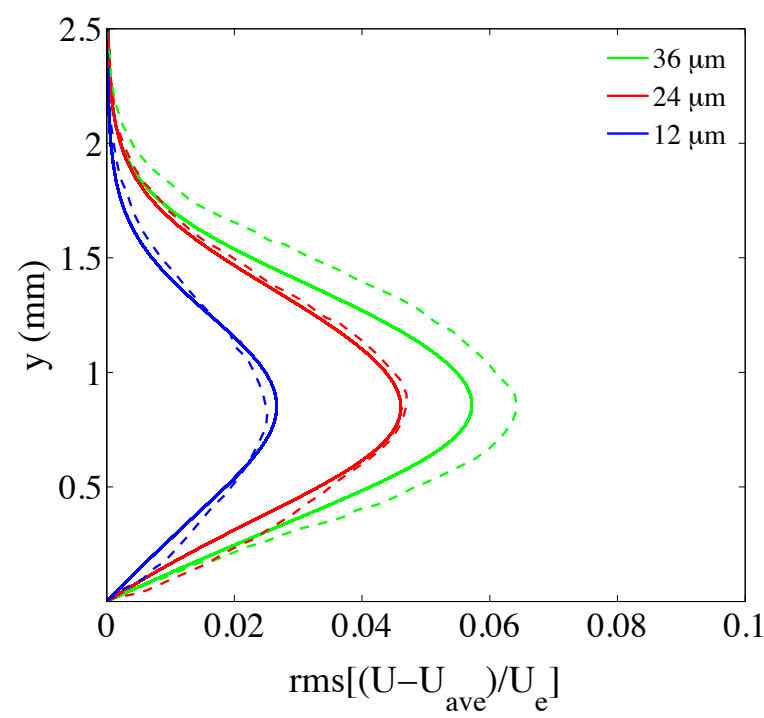

(a) Stationary crossflow mode shape

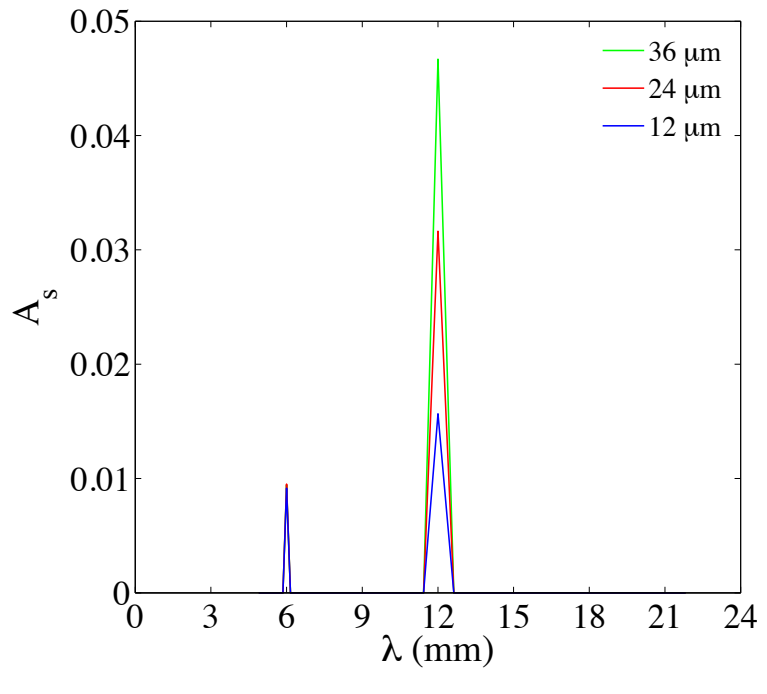

(b) Amplitude vs wave number for spanwise signal at maximum amplitude

Figure 13. Stationary mode shape and amplitude spectra at $20 \% x / c, \lambda=12 \mathrm{~mm}$ (Roughness height, k, indicated on graph) 


\section{Receptivity of DRE Array at Control Wavelength Results}

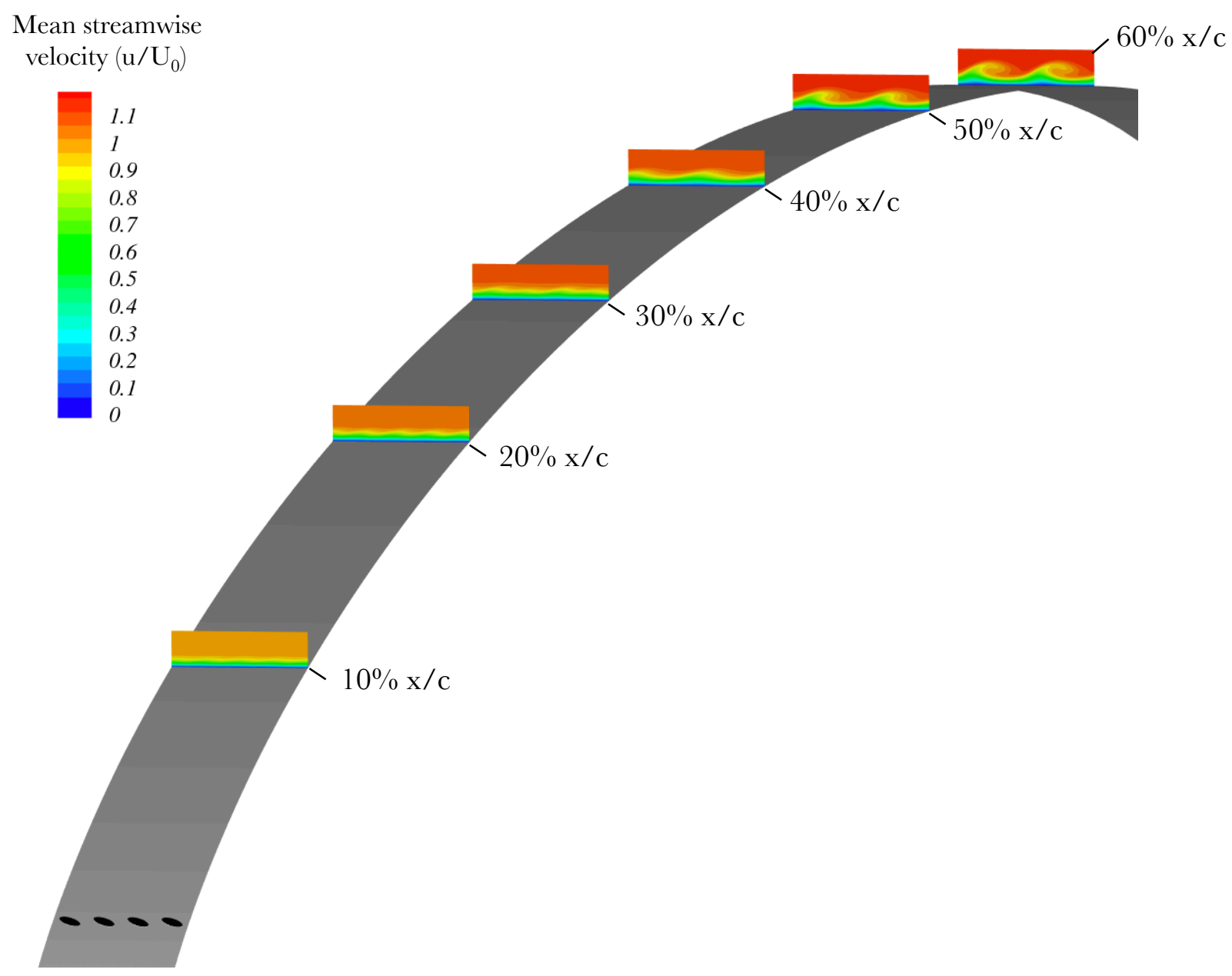

Figure 14. Contours of mean streamwise velocity $u / U_{0}, k=42 \mu m, \lambda=6 \mathrm{~mm}$, Contours taken at $x / c=$ $10 \%, 20 \%, 30 \%, 40 \%, 50 \%, 60 \%$

Simulation results were obtained for modelling of roughness arrays at the control wavelength $6 \mathrm{~mm}$. Figure 14 shows an overview of the flowfield for the $6 \mathrm{~mm}$ wavelength roughness array and $k=42 \mu \mathrm{m}$. At $20 x / c$ the disturbance can be clearly visualised and a spanwise periodicity with a clear $6 \mathrm{~mm}$ wavelength. At 30 $x / c$ however a $12 \mathrm{~mm}$ mode appears dominant resulting in 2 clear crossflow vortex formations by $50 \% x / c$. The experiment by Hunt and Saric ${ }^{1}$ used a naphthalene flow visualisation method to visualise the transition locations. They report that the transition location does not move downstream at the control wavelength but in fact moved the transition location upstream. A cause for this can be attributed to the size of the roughness elements being too large and the $12 \mathrm{~mm}$ mode is not suppressed, clearly shown in Figure 14 .

For the control wavelength the experimental test case measured the receptivity of the roughness element arrays by applying hot-wire measurement scans at $15 \% x / c$. Figure 15 shows contours of mean flow streamwise velocity $\left(u / U_{e}\right)$ at $15 \% x / c$ for the three roughness element heights. The figures on the left show the experimental hot-wire scans while the figures on the right show the simulation results. As with the critical wavelength results the hot-wire scans were taken with a $1 \mathrm{~mm}$ spacing resulting in 65 locations across the span. The simulation however contains 156 spanwise locations across a span of $24 \mathrm{~mm}$. The larger roughness element sizes $(k=27 \mu \mathrm{m}$ and $k=42 \mu \mathrm{m})$ show a spanwise periodic signal with a clear $6 \mathrm{~mm}$ wavelength and a good resemblance to the experimental data. The $k=14 \mu \mathrm{m}$ case however features variations in amplitude for each disturbance across the span, suggesting that the initial amplitude was not captured accurately and that the grid may be too coarse near the cylinder for the smallest roughness height to capture the exact initial amplitude. 
Figure 16 shows mean flow streamwise velocity profiles taken from the contours shown in Figure 15. The mean boundary layer profile is displayed in the figure and coloured red. Figure 17 shows the subsequent disturbance velocity profiles obtained by removing the mean of the individual profiles away from each individual profiles. For both figures the experimental plots are displayed on the left while the simulation results are displayed on the right. The simulation results show a good agreement with the experimental plots; the size and shape of the disturbance is accurately modelled along with the pattern of s-shaped profiles.

The stationary mode shape for the $6 \mathrm{~mm}$ wavelength at $15 \% \mathrm{x} / \mathrm{c}$ is shown in Figure 18(a) and the maximum modal decomposition amplitude plot in Figure 18(b). The stationary mode shape for the experiment shows a primary lobe centred at $0.9 \mathrm{~mm}$ and a smaller secondary lobe at $0.2 \mathrm{~mm}$. The simulations capture the primary lobe and the amplitude very good accuracy for the $k=27 \mu \mathrm{m}$ and $k=42 \mu \mathrm{m}$ cases. However the $k=14 \mu m$ case over-predicts the amplitude by $33 \%$, similar over prediction to the results of the smallest roughness height obtained for the critical wavelength. The secondary lobe in the lower part of the boundary layer however is not captured by the simulations, the secondary lobe may have been a remnant of the coarse resolution of the hot wire measurements. The amplitude plot in Figure 18(a) shows a predominant $6 \mathrm{~mm}$ mode at this location with their amplitude close to the overall disturbance amplitude.

Overall the results from both the critical and control spanwise wavelengths show good agreement to the experiment using the modified grid and the LES captures the flow features well. As no secondary freestream perturbations are introduced the transition location cannot be compared. Further work from these results will attempt to introduce a realistic freestream turbulence environment to model the entire transition process (receptivity to breakdown). 

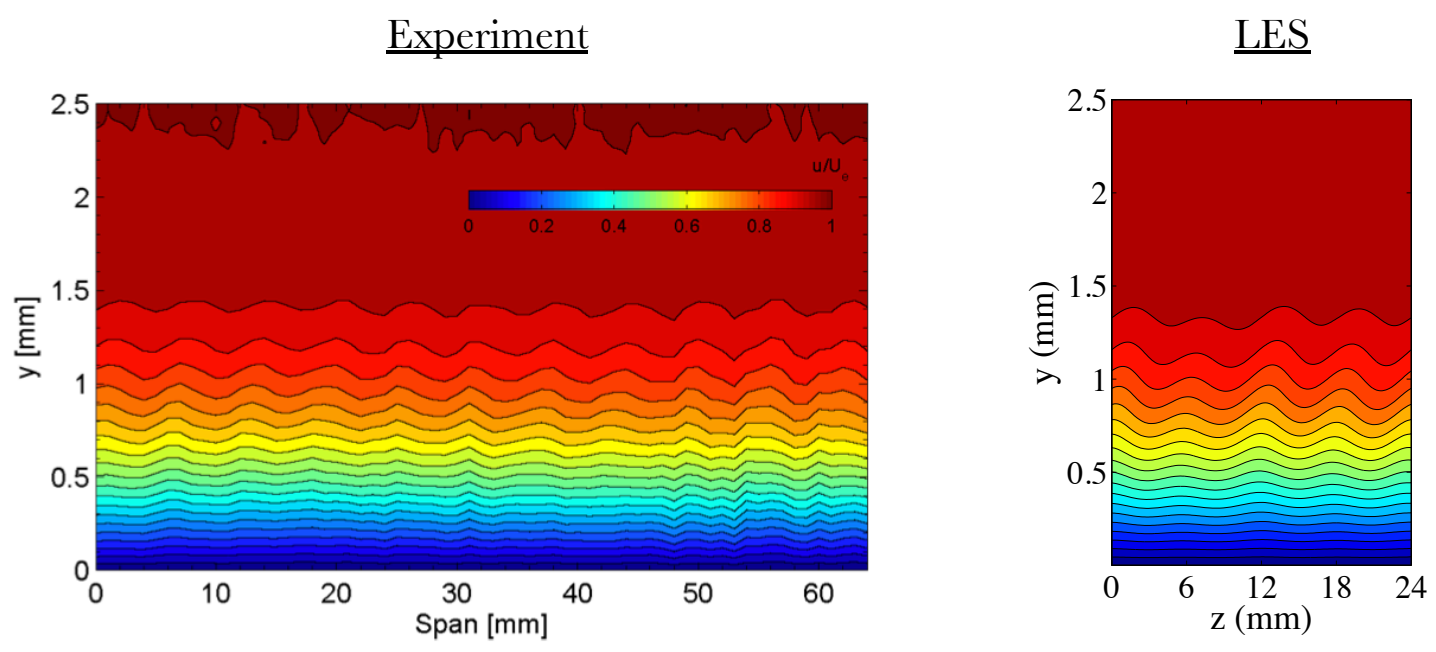

(a) $k=14 \mu m, \lambda=6 m m$
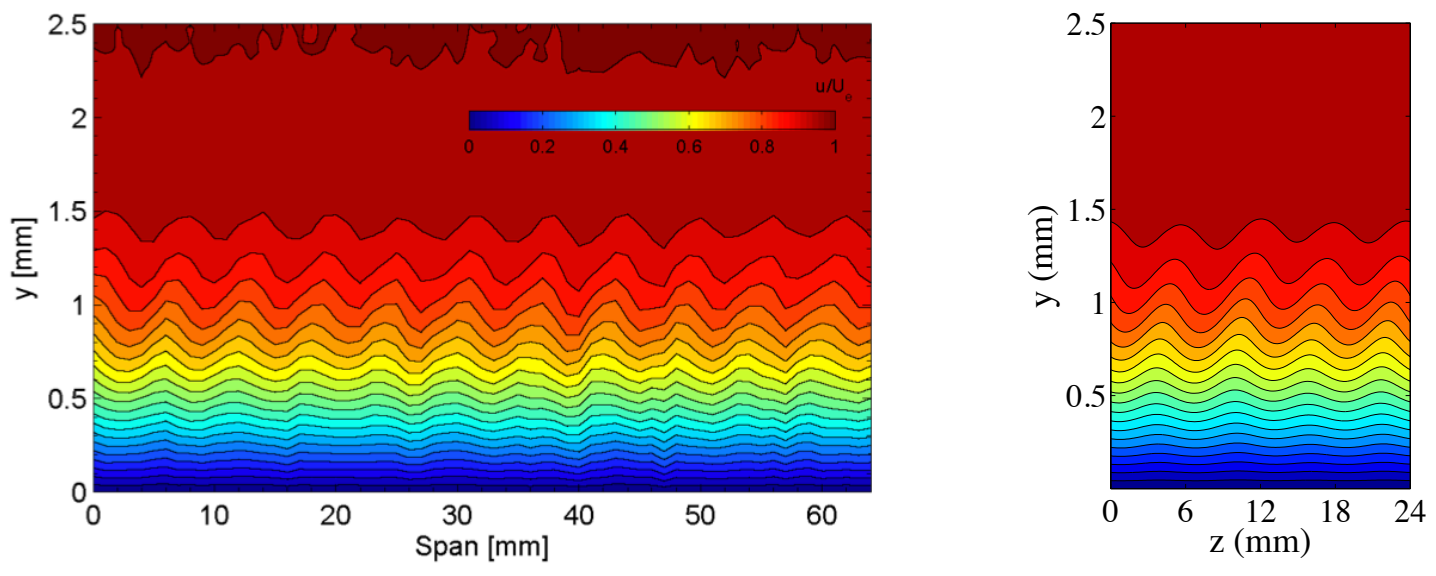

(b) $k=27 \mu m, \lambda=6 m m$
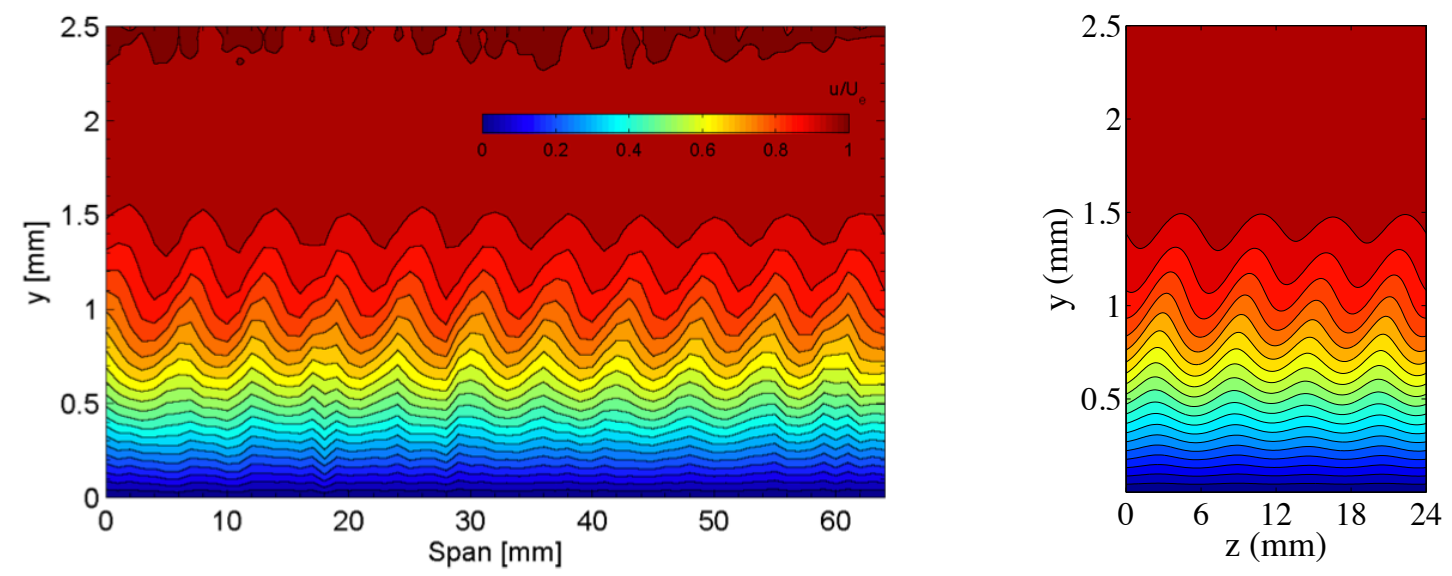

(c) $k=42 \mu m, \lambda=6 m m$

Figure 15. Contours of streamwise velocity $\left(u / U_{e}\right)$ at $15 \% x / c$ Left: Experimental (Hunt and Saric $\left.{ }^{1}\right)$ Right: LES 
$\underline{\text { Experiment }}$

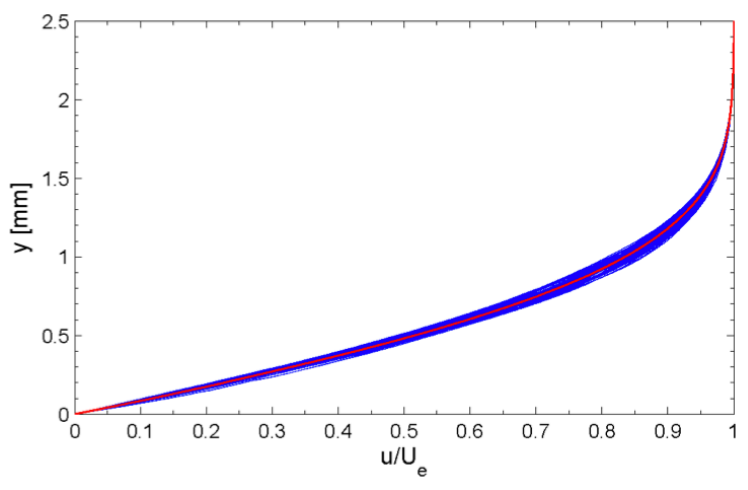

$\underline{\text { LES }}$

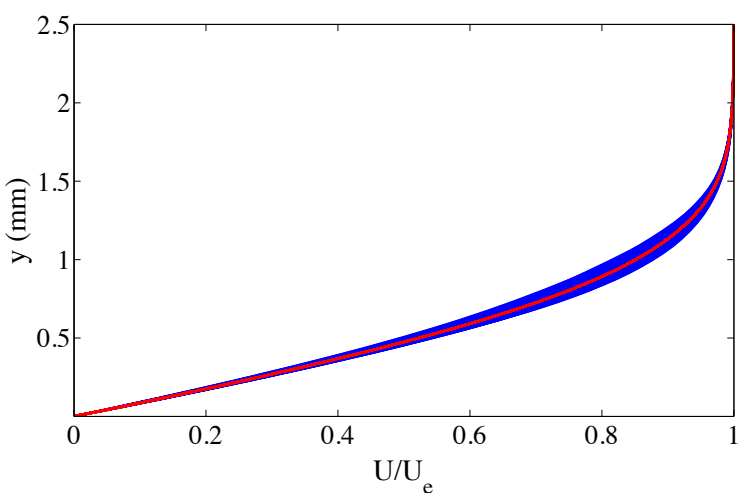

(a) $k=14 \mu m, \lambda=6 m m$
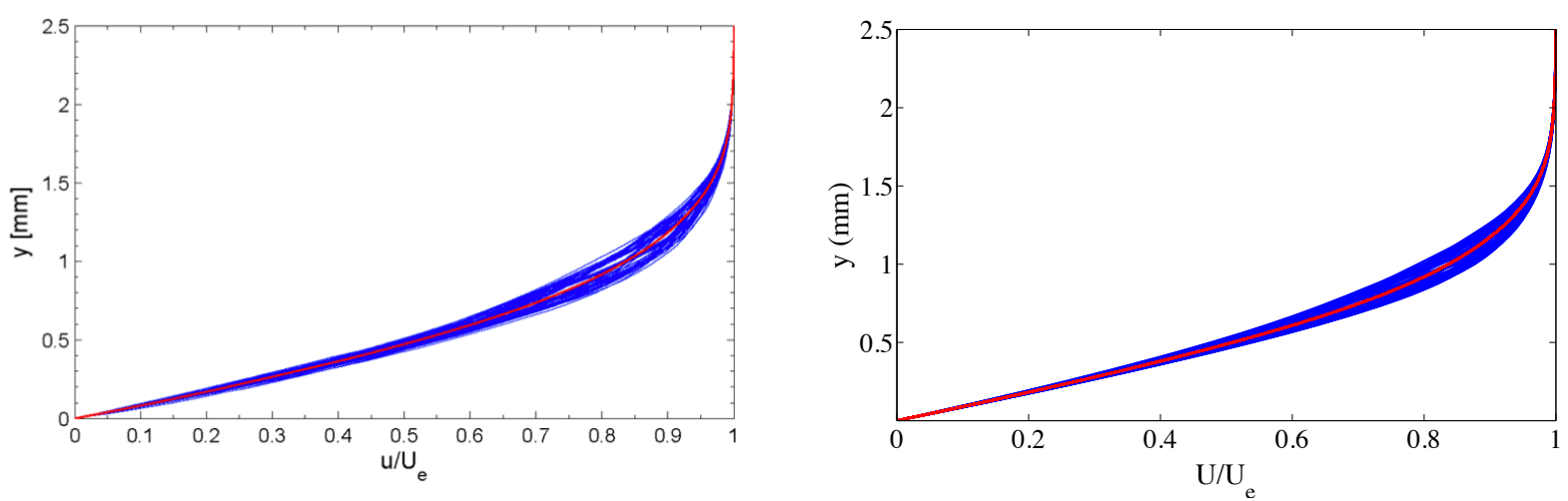

(b) $k=27 \mu m, \lambda=6 m m$

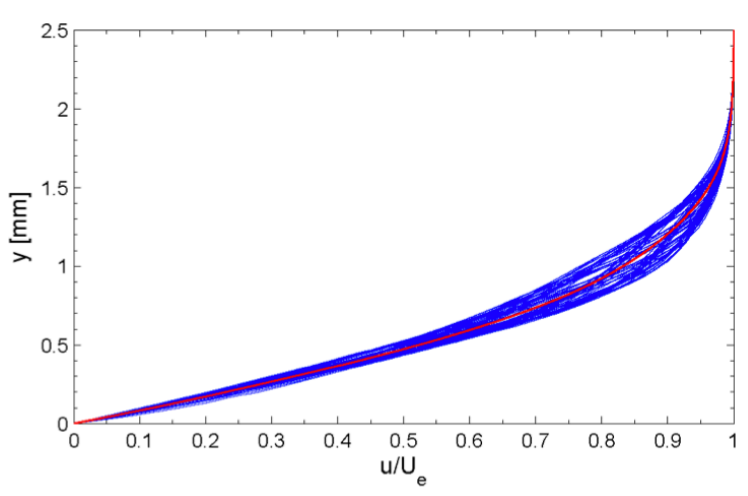

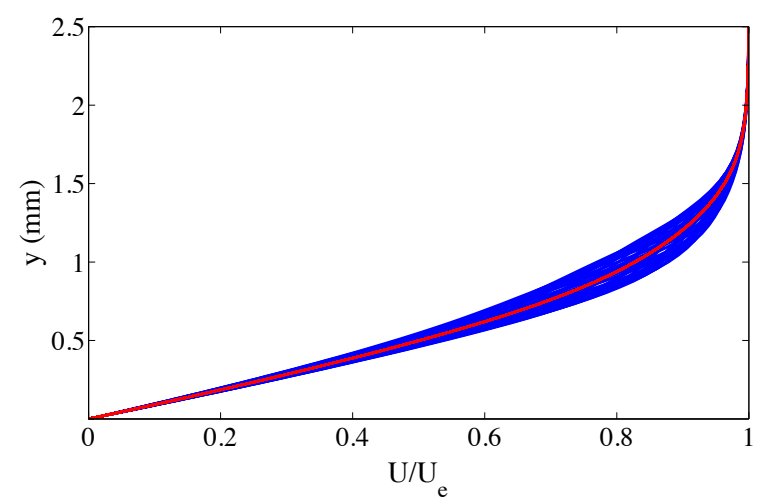

(c) $k=42 \mu m, \lambda=6 m m$

Figure 16. Spanwise array of mean-flow boundary-layer profiles across span at $15 \% x / c$, The mean of the profiles is displayed in red, Left: Experimental (65mm span) (Hunt and Saric ${ }^{1}$ ) Right: LES (24mm span) 
$\underline{\text { Experiment }}$

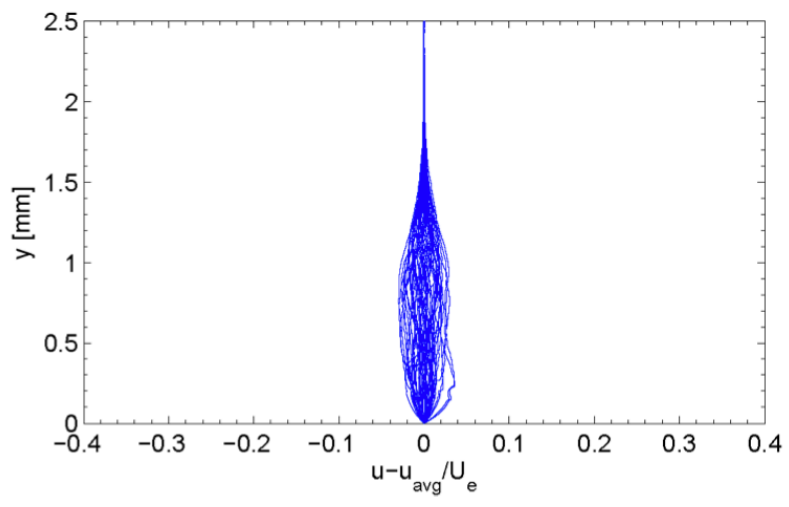

$\underline{\text { LES }}$

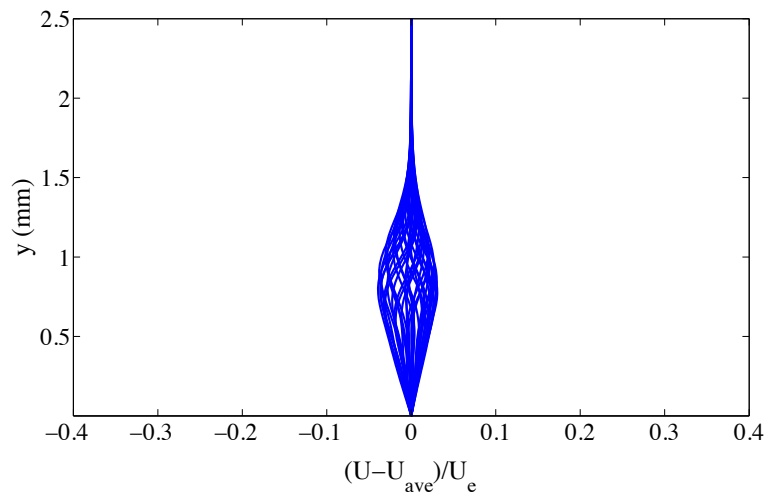

(a) $k=14 \mu m, \lambda=6 m m$
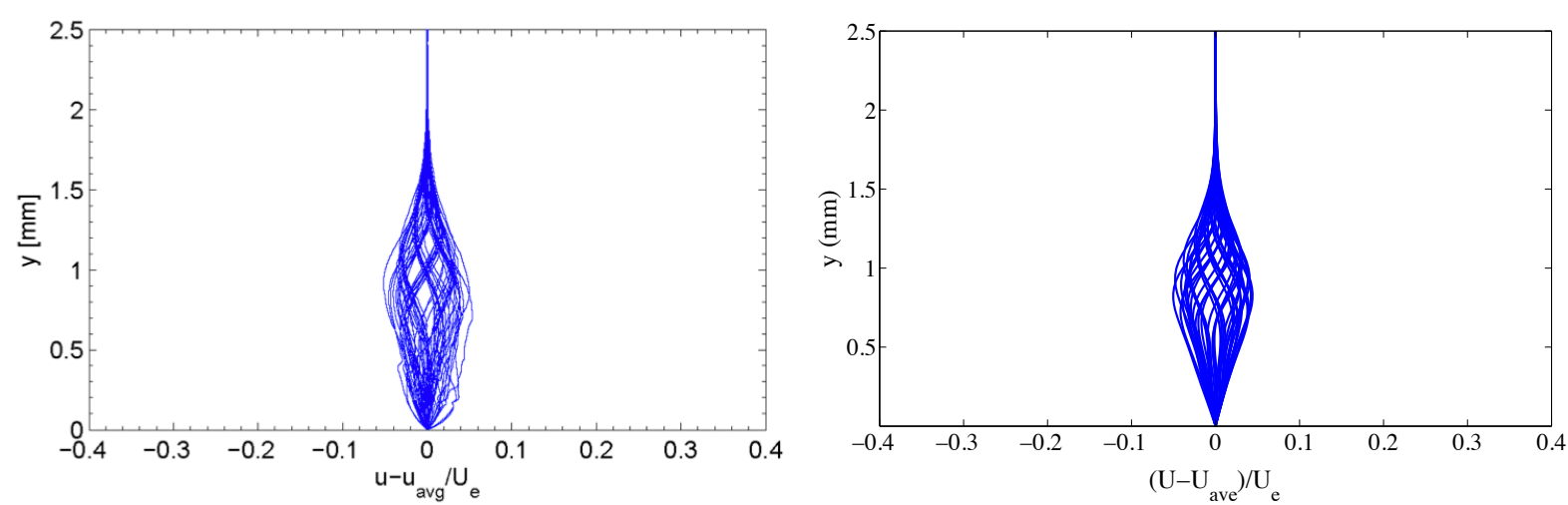

(b) $k=27 \mu m, \lambda=6 m m$
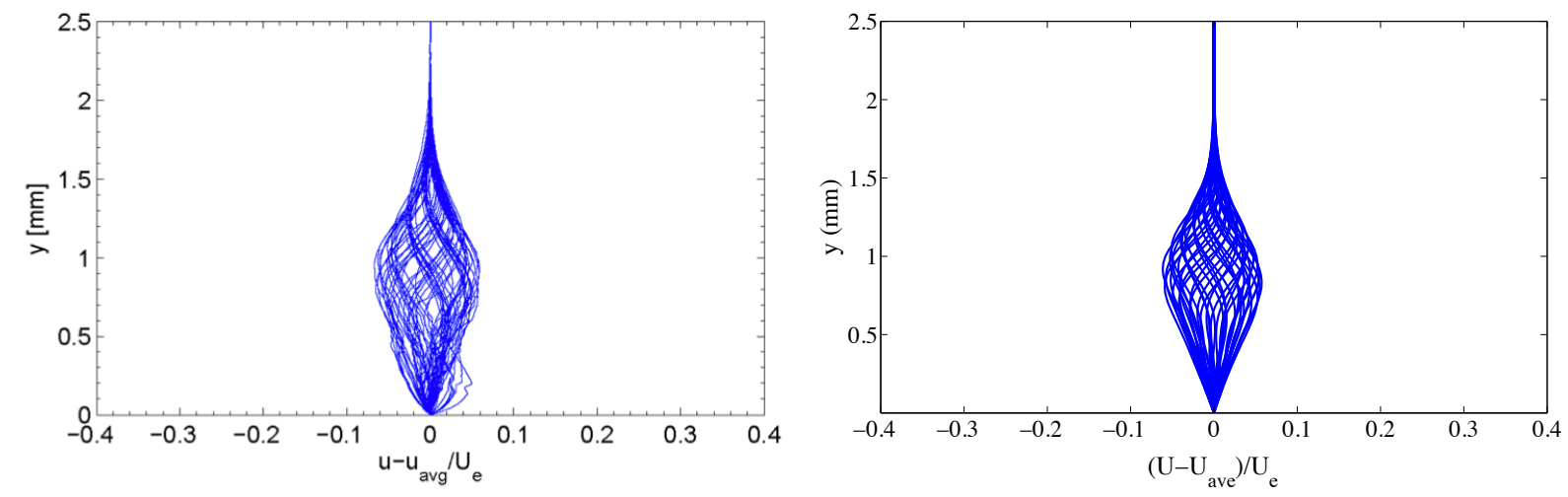

(c) $k=42 \mu m, \lambda=6 m m$

Figure 17. Spanwise array of disturbance velocity profiles across span at $15 \% x / c$ Left: Experimental (65mm span) (Hunt and Saric ${ }^{1}$ ) Right: LES (24mm span) 


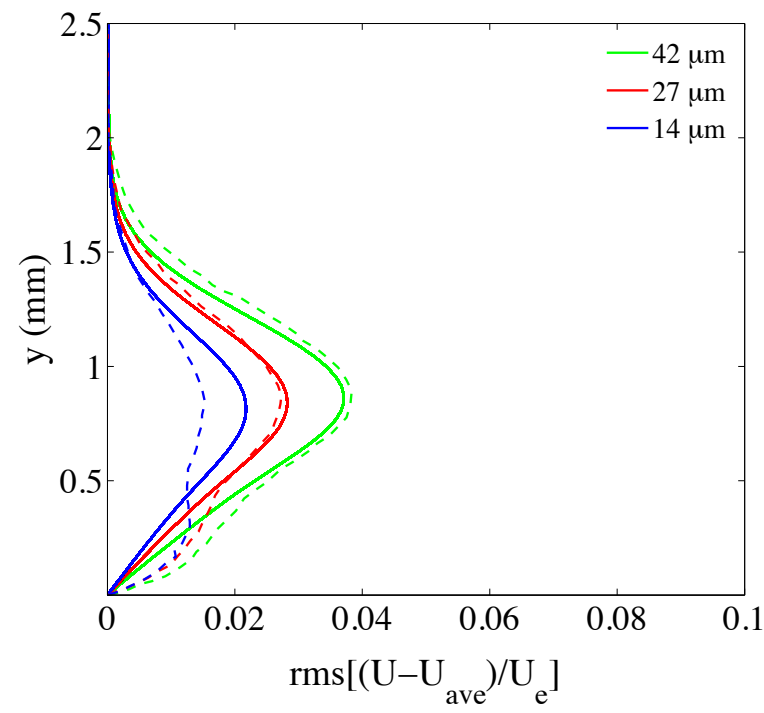

(a) Stationary crossflow mode shape

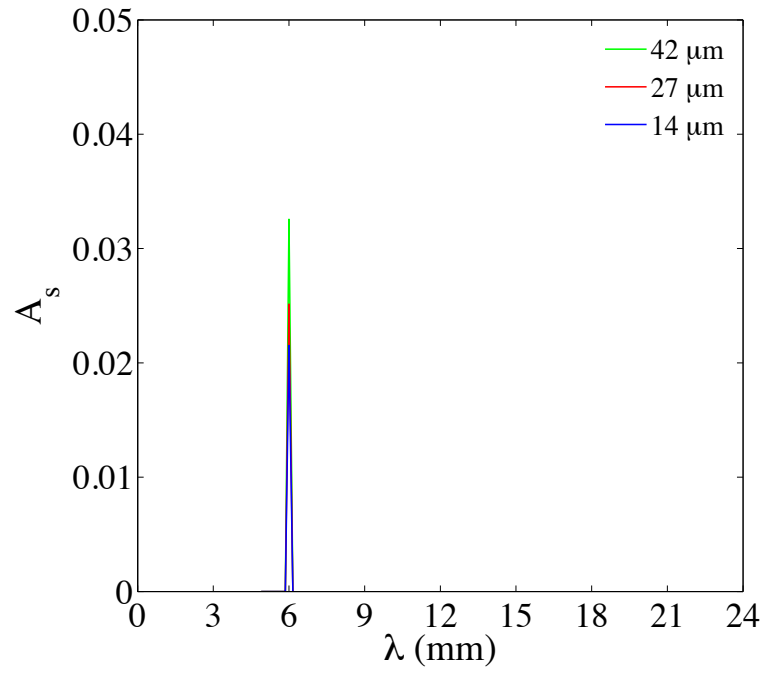

(b) Amplitude vs wave number for spanwise signal at maximum amplitude

Figure 18. Stationary mode shape and amplitude spectra at $15 \% x / c, \lambda=6 \mathrm{~mm}$ (Roughness height, k, indicated on graph)

\section{Conclusions}

The aim of the paper was to model arrays of roughness elements using a highly resolved large eddy simulation method and to compare the solutions to that of the experiments from Hunt and Saric. ${ }^{1}$ A secondary purpose for the paper was to understand the flow around the cylinder and to determine the grid resolution requirements for properly capturing the disturbance. This was accomplished for a range of roughness heights at two spanwise wavelengths: a critical case $(\lambda=12 \mathrm{~mm})$ and a control case $(\lambda=6 \mathrm{~mm})$.

An initial and modified grid were developed to study the effect of grid spacing in capturing the disturbance around each roughness element. The initial grid failed to capture the disturbance accurately and further grid refinement was required in the immediate vicinity around the cylinder. The cylinders disturbed the flow up to $1 / 5$ of the cylinder diameter $(0.6 \mathrm{~mm})$ away from the cylinder and this region must be resolved carefully. For the modified grid in this paper the region was capturing using 10 computational grid nodes. Streamlines around the cylinder showed how the flow deflects around the roughness element with a disturbance in the spanwise velocity of up to $0.02 Q_{0}$.

For the critical wavelength case $(\lambda=12 \mathrm{~mm})$ results were obtained at roughness heights $k=12,24$, and $36 \mu \mathrm{m}$. The simulations were compared to experimental results at $15 \% x / c$ and $20 \% x / c$. The simulations compared well with the experimental data, the stationary mode shapes showed excellent agreement in terms of size, shape and amplitude of the disturbance. Decomposition of the spanwise signal showed a predominant $12 \mathrm{~mm}$ mode with shorter peaks at $6 \mathrm{~mm}$. The overall view of the flow field showed the $12 \mathrm{~mm}$ mode dominate to form saturated crossflow vortices.

For the control wavelength case $(\lambda=6 \mathrm{~mm})$ results were obtained at roughness height $k=14,27$, and $42 \mu \mathrm{m}$. The simulations were compared to experimental results at $15 \% x / c$. Much like the critical wavelength case the simulations successfully captured the disturbance to a good agreement with the experimental data. The $6 \mathrm{~mm}$ mode dominates the spanwise signal and a clear spanwise uniformity is shown. The amplitudes match well with the experimental data however the smallest roughness height $(k=14 \mu \mathrm{m}$ over predicts the amplitude by $33 \%$. This cause of this could be that for this roughness height the grid may require addition nodes, or could be attributed to variations in roughness element shape and size in the experiments. The overall flow field showed the $6 \mathrm{~mm}$ mode decay by $30 \% \mathrm{x} / \mathrm{c}$ and the critical wavelength begin to grow.

The overall conclusions that are made from the paper are that the numerical method described can successfully capture the receptivity of roughness arrays and can be used in further calculations to model 
transition location. For the results presented in this paper no secondary perturbations were generated as the secondary instability and transition region were not the focus. The benefit of using a method demonstrated in this paper is that it is capable of modelling the full process of transition (receptivity, primary growth, secondary instabilities and transition) with less computational resources required than a DNS, giving it potential to be used for design and on more realistic flow conditions in future work.

\section{Acknowledgements}

The author would like to thank the Flight Physics department at Airbus, Filton for their provision of supercomputing time using the ASRC facilities \& financial support for the project. I would also like to thank HPC Midlands for time on the Hera super computer.

\section{References}

${ }^{1}$ L.E. Hunt. Boundary-Layer Receptivity to Three-Dimensional Roughness Arrays on a Swept-Wing. PhD thesis, Texas A\&M University, December 2011.

${ }^{2}$ Airbus. Airbus Global Market Survey 2011-2030, September 2011.

${ }^{3}$ ACARE. European Aeronautics: A vision for 2020, July 2001.

${ }^{4}$ High level group on aviation research. Flightpath 2050: Europe's vision for Aviation, July 2011.

${ }^{5}$ G Schrauf. Status and perspectives of laminar flow. Aeronautical Journal, 109(1102):639-644, 2005.

${ }^{6}$ Pfenninger, W. Some observations of the transition process on the windward face of a long yawed cylinder. Recent Developments in Boundary Layer Research, AGARDograf 97, 1965.

${ }^{7}$ D. I. A. Poll. Some Observations of the Transition Process on the Windward Face of a Long Yawed Cylinder. Journal of Fluid Mechanics, 150:329-356, 1985.

${ }^{8}$ E. B. White and W. S Saric. Secondary instability of crossflow vortices. Journal of Fluid Mechanics, 525:275-308, 2005.

${ }^{9}$ William S Saric, Helen L Reed, and Edward B White. Stability and Transition of Three-Dimensional Boundary Layers. Annual Review of Fluid Mechanics, 35(1):413-440, January 2003.

${ }^{10} \mathrm{H}$. Bippes. Environmental conditions and transition prediction in 3-D boundary layers. AIAA 97-1906, 1997.

${ }^{11} \mathrm{H}$. Deyhle and H. Bippes. Disturbance growth in an unstable three-dimensional boundary layer and its dependence on initial conditions. Journal of Fluid Mechanics, 316:73-113, 1996.

${ }^{12}$ Wasserman, P. and Kloker, M. Transition mechanism in a 3D boundary layer with pressure gradient changeover. Journal of Fluid Mechanics, 530:265, 2005.

${ }^{13}$ W. S. Saric, R. B. Carrillo, and M. S. Reibert. Leading-edge roughness as a transition control mechanism. AIAA Pap. No. 98-0781, 1998.

${ }^{14}$ M. R. Malik, F. Li, M. M. Choudhari, and C.-L. Chang. Secondary instability of crossflow vortices and swept-wing boundary-layer transition. Journal of Fluid Mechanics, 399:85-115, 1999.

${ }^{15}$ A. Carpenter, W. S. Saric, and H. L. Reed. Laminar flow control on a swept wing with distributed roughness. AIAA Pap. No. 2008-7335, 2008.

${ }^{16}$ Wasserman, P. and Kloker, M. Transition mechanisms induced by travelling crossflow vortices in a three-dimensional boundary layer. Journal of Fluid Mechanics, 483:67-89, 2003.

${ }^{17}$ D. Tempelmann, L. U. Schrader, A. Hanifi, L. Brandt, and D. S. Henningson. Swept wing boundary-layer receptivity to localised surface roughness. Journal of Fluid Mechanics, 711:516-544, 2011.

${ }^{18}$ M. S. Reibert, W. S. Saric, R. B. Carrillo, and K. L. Chapman. Experiments in nonlinear saturation of stationary crossflow vortices in a swept-wing boundary layer. AIAA Pap. No. 96-0184, 1996.

${ }^{19}$ S. M. Hosseini, D. Tempelmann, A. Hanifi, and D. S. Henningson. Stabilization of a swept-wing boundary layer by distributed roughness elements. Journal of Fluid Mechanics Rapids, 718:R1, 2013.

${ }^{20} \mathrm{H}$ Salman, D Jiang, J. J. McGuirk, and G. J. Page. Linear and non-linear turbulence model predictions of vortical flows in lobed mixers. Aeronautical Journal, (108):65-73, 2004.

${ }^{21}$ G. J. Page, Q. Li, and J. J. McGuirk. LES of Impinging Jet Flows Relevant to Vertical Landing Aircraft. In The 23rd Applied Aerodynamics Conference, Toronto, Canada, 2005.

${ }^{22}$ R. E. Rife and G. J. Page. Large Eddy Simulation of high Reynolds number jets with microject injection. In $17 t h$ AIAA/CEAS Aeroacoustics Conference, Portland, Oregon, 2011.

${ }^{23} \mathrm{~J}$. Smagorinsky. General circulation experiments with the primitive equations I. The basic experiment. Monthly Weather Review, 91:99-164, 1963.

${ }^{24}$ E. R. Van Driest. On turbulent flow near a wall. Journal of the Aeronautical Sciences, 23:1007-1011, 1956.

${ }^{25}$ M. Piomelli Germano, P. U. Moin, and W. H. Cabot. A dynamic subgrid-scale eddy-viscosity model. Physics of Fluids A: Fluid Dynamics, 3(1760), 1991.

${ }^{26}$ F. Nicoud and F. Ducros. Subgrid-Scale Stress Modelling Based on the Square of the Velocity Gradient Tensor. Flow, Turbulence and Combustion, 62:183-200, 1999.

${ }^{27}$ V. I. Mistry, G. J. Page, and McGuirk J. J. Large eddy simulation of crossflow vortices on an infinite swept wing. In 42nd AIAA Fluid Dynamics Conference and Exhibit, 25 - 28 June 2012, New Orleans, Louisiana.

${ }^{28}$ U. Piomelli and E. Balaras. Wall-layer models for large-eddy simulations. Annual Review Of Fluid Mechanics, 34:349-374, 2002. 